MATHEMATICS OF COMPUTATION

Volume 68, Number 227, Pages 1149-1178

S 0025-5718(99)01042-X

Article electronically published on February 10, 1999

\title{
DOUBLE ROOTS OF $[-1,1]$ POWER SERIES AND RELATED MATTERS
}

\author{
CHRISTOPHER PINNER
}

\begin{abstract}
For a given collection of distinct arguments $\vec{\theta}=\left(\theta_{1}, \ldots, \theta_{t}\right)$, multiplicities $\vec{k}=\left(k_{1}, \ldots, k_{t}\right)$, and a real interval $I=[U, V]$ containing zero, we are interested in determining the smallest $r$ for which there is a power series $f(x)=1+\sum_{n=1}^{\infty} a_{n} x^{n}$ with coefficients $a_{n}$ in $I$, and roots $\alpha_{1}=r e^{2 \pi i \theta_{1}}, \ldots, \alpha_{t}=r e^{2 \pi i \theta_{t}}$ of order $k_{1}, \ldots, k_{t}$ respectively. We denote this by $r(\vec{\theta}, \vec{k} ; I)$.

We describe the usual form of the extremal series (we give a sufficient condition which is also necessary when the extremal series possesses at least $\left(\sum_{i=1}^{t} \delta\left(\theta_{i}\right) k_{i}\right)-1$ non-dependent coefficients strictly inside $I$, where $\delta\left(\theta_{i}\right)$ is 1 or 2 as $\alpha_{i}$ is real or complex).

We focus particularly on $r(\theta, 2 ;[-1,1])$, the size of the smallest double root of a $[-1,1]$ power series lying on a given ray (of interest in connection with the complex analogue of work of Boris Solomyak on the distribution of the random series $\left.\sum \pm \lambda^{n}\right)$. We computed the value of $r(\theta, 2 ;[-1,1])$ for the rationals $\theta$ in $(0,1 / 2)$ of denominator less than fifty. The smallest value we encountered was $r(4 / 29,2 ;[-1,1])=0.7536065594 \ldots$ For the onesided intervals $I=[0,1]$ and $[-1,0]$ the corresponding smallest values were $r(11 / 30,2 ;[0,1])=.8237251991 \ldots$ and $r(1 / 3,2 ;[-1,0])=.8656332072 \ldots$.
\end{abstract}

\section{INTRODUCTION}

For a fixed, closed, interval $I:=[U, V]$ containing zero, we let $\mathcal{F}_{I}$ denote the set of real power series

$$
\mathcal{F}_{I}:=\left\{1+\sum_{n=1}^{\infty} a_{n} x^{n}: a_{n} \in I\right\} .
$$

For a vector of arguments $\vec{\theta}=\left(\theta_{1}, \ldots, \theta_{t}\right)$ and a vector of multiplicities $\vec{k}=$ $\left(k_{1}, \ldots, k_{t}\right)$, we let $\mathcal{R}(\vec{\theta}, \vec{k} ; I)$ denote the set of positive real numbers, $r$, for which there is a series in $\mathcal{F}_{I}$ with roots at $\alpha_{1}=r e^{2 \pi i \theta_{1}}, \ldots, \alpha_{t}=r e^{2 \pi i \theta_{t}}$, of multiplicity $k_{1}, \ldots, k_{t}$ respectively, and, when $\mathcal{R}(\vec{\theta}, \vec{k} ; I)$ is non-empty, we define $r(\vec{\theta}, \vec{k} ; I)$ to be the infimum of this set. By a $k$ th order root of $f(x)$ we mean of course an $\alpha$ such that $f(\alpha)=f^{\prime}(\alpha)=\cdots=f^{(k-1)}(\alpha)=0$. By symmetry $x \mapsto \bar{x}$ we can clearly restrict our attention to $0 \leq \theta_{i} \leq 1 / 2$ (and in the case of a single argument and symmetric intervals $I$ to $0 \leq \theta \leq 1 / 4$, from $x \mapsto-x$ ).

In [2] we computed the smallest simple root on a single ray, Boris Solomyak [5] having previously considered this problem for $[0,1]$ power series. In [1] we computed

Received by the editor July 12, 1996 and, in revised form, November 7, 1997.

1991 Mathematics Subject Classification. Primary 30C15; Secondary 30B10, 12D10.

Key words and phrases. Power series, restricted coefficients, double roots.

(C)1999 American Mathematical Society 
the smallest $k$ th order real root of a $[-1,1]$ power series for $k \leq 27$ (the real case necessarily gives the smallest $k$ th order root). Solomyak [6] had in fact earlier shown that the smallest double real root of a $[-1,1]$ power series is $\approx .6491$, which he used to show that the distribution of the random series $\sum_{n=0}^{\infty} \pm \lambda^{n}$ (where the plus and minus signs are chosen independently, each with a probability $1 / 2$ ) has $L^{2}$ density for a.e. $\lambda$ in $(1 / 2,1)$. In a private communication he conjectured that the smallest complex double root of a $[-1,1]$ power series was substantially larger than the smallest double real root, in particular suggesting that it was probably of absolute value at least $1 / \sqrt{2}$, which would have implications for the complex analogue of his result. We thus focus our computational attention on $r(\theta, 2 ;[-1,1])$, the size of the smallest double root of a $[-1,1]$ power series lying on a given ray. Our computations of the value of $r(\theta, 2 ;[-1,1])$ for rational $\theta$ of denominator less than fifty suggest that that the minimum value in the complex case is actually close to $r(4 / 29,2 ;[-1,1])=.7536065594 \ldots$. We similarly computed the double zero free region for power series with coefficients in the one-sided intervals $[0,1]$ and $[-1,0]$. The smallest complex double zeros we encountered in those cases were of size $r(11 / 30,2 ;[0,1])=.8237251991 \ldots$ and $r(1 / 3,2 ;[-1,0])=.8656332072 \ldots$ (the latter is probably the exact minimum). Somewhat related problems on double zeros of $\{0,1\}$ power series occur in the work of Odlyzko \& Poonen [4]. Here we have concentrated on the more manageable class of power series with coefficients in intervals. Unfortunately the extremal power series in our situation almost certainly have some non-integer coefficients, integer analogues remaining unclear.

We let

$$
\delta(\theta):= \begin{cases}1 & \text { if } e^{2 \pi i \theta} \text { is real } \\ 2 & \text { if } e^{2 \pi i \theta} \text { is complex }\end{cases}
$$

and define

$$
M=M(\vec{\theta}, \vec{k}):=\sum_{i=1}^{t} \delta\left(\theta_{i}\right) k_{i}
$$

We first note that crude bounding ensures that $r(\vec{\theta}, \vec{k} ; I)<1$ in all cases of interest.

Lemma 1. Suppose that $U<0$ and $V>0$. Let $N:=\left\lceil 2^{M+1} / \min \{|U|,|V|\}\right\rceil$, and let $p$ denote the $N$ th prime greater than $M=M(\vec{\theta}, \vec{k})$. Then

$$
r(\vec{\theta}, \vec{k} ;[U, V]) \leq \frac{1}{2^{1 / p M}} .
$$

If none of the $\theta_{i}=0$, then

$$
r(\vec{\theta}, \vec{k} ;[0, V])<1, \quad r(\vec{\theta}, \vec{k} ;[U, 0])<1 .
$$

The exclusion of $\theta_{i}=0$ in the case of totally positive or totally negative intervals is plainly necessary (by Descartes' rule of signs a power series cannot have a $k$ th order positive real root without at least $k$ sign changes in the coefficients). It appears from our computations that $r(\theta, 2 ;[-1,1]) \leq r(1 / 4,2 ;[-1,1])=$ $\sqrt{r(0,2 ;[-1,1])}=.8056909214 \ldots$ Notice that we also have an angle-independent lower bound $r(\vec{\theta}, \vec{k} ; I) \geq r\left(0, \max k_{i} ;[-A, A]\right), A=\max \{|U|,|V|\}$, from considering the real part of $f\left(x e^{2 \pi i \theta}\right)$.

Since we are thus working safely inside the unit circle, an appeal to compactness ensures that for our purposes $r(\vec{\theta}, \vec{k} ; I)$ is always achieved. 
For a vector of arguments $\vec{\theta}$, multiplicities $\vec{k}$ and a vector of non-negative integers $\vec{n}=\left(n_{1}, \ldots, n_{M}\right)$, we define the $\delta\left(\theta_{j}\right) k_{j} \times M$ submatrices

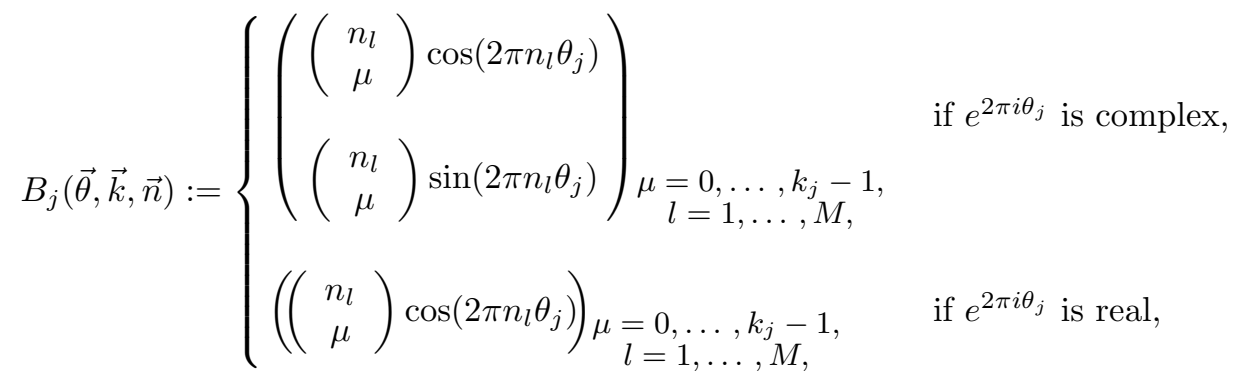

and the resulting $M \times M$ matrix

$$
B(\vec{\theta}, \vec{k}, \vec{n}):=\left(\begin{array}{c}
B_{1}(\vec{\theta}, \vec{k}, \vec{n}) \\
\vdots \\
B_{t}(\vec{\theta}, \vec{k}, \vec{n})
\end{array}\right) .
$$

For a vector of positive integers $\vec{N}=\left(N_{1}, \ldots, N_{M-1}\right)$, and a non-negative integer $n$, we define

$$
\vec{N}_{n}:=\left(n, N_{1}, \ldots, N_{M-1}\right)
$$

We let

$$
\mathcal{S}(\vec{\theta}, \vec{k}, \vec{N}):=\left\{n \in \mathbf{N}: \operatorname{det} B\left(\vec{\theta}, \vec{k}, \vec{N}_{n}\right)=0\right\}
$$

It will be convenient to write our interval $I$ (always assumed to contain zero) in the form

$$
I:=[B-A, B+A]
$$

We prove the following structure theorem for the power series corresponding to the extremal root:

Theorem 1. Let $F(x)=1+\sum_{i=0}^{\infty} a_{i} x^{i}$ denote a power series in $\mathcal{F}_{I}$ with roots of order $k_{1}, \ldots, k_{t}$ at $\alpha_{1}=r e^{2 \pi i \theta_{1}}, \ldots, \alpha_{t}=r e^{2 \pi i \theta_{t}}$ with $r=r(\vec{\theta}, \vec{k} ; I)$. If there exists a vector of $M(\vec{\theta}, \vec{k})-1$ positive integers $\vec{N}=\left(N_{1}, \ldots, N_{M-1}\right)$ such that $a_{N_{1}}, \ldots, a_{N_{M-1}}$ all lie strictly inside $I$, then either $\operatorname{det} B\left(\vec{\theta}, \vec{k}, \vec{N}_{n}\right)=0$ for all $n$, or $\operatorname{det} B\left(\vec{\theta}, \vec{k}, \vec{N}_{0}\right) \neq 0$ and the remaining coefficients of this extremal power series satisfy

$$
a_{n}=B-\operatorname{sign}\left(\frac{\operatorname{det} B\left(\vec{\theta}, \vec{k}, \vec{N}_{n}\right)}{\operatorname{det} B\left(\vec{\theta}, \vec{k}, \vec{N}_{0}\right)}\right) A, \quad \text { for } n \notin \mathcal{S}(\vec{\theta}, \vec{k}, \vec{N}) .
$$

Conversely if the series $f(x)=1+\sum_{i=1}^{\infty} c_{i} x^{i}$ in $\mathcal{F}_{I}$ has a root of order $k_{j}$ at $\alpha_{j}=r e^{2 \pi i \theta_{j}}$ for $j=1, \ldots, t$, and coefficients such that for some vector of $(M-1)$ positive integers $\vec{N}$ with $\operatorname{det} B\left(\vec{\theta}, \vec{k}, \vec{N}_{0}\right) \neq 0$

$$
c_{n}=B-\operatorname{sign}\left(\frac{\operatorname{det} B\left(\vec{\theta}, \vec{k}, \vec{N}_{n}\right)}{\operatorname{det} B\left(\vec{\theta}, \vec{k}, \vec{N}_{0}\right)}\right) A, \quad \text { for } n \notin \mathcal{S}(\vec{\theta}, \vec{k}, \vec{N}),
$$

then $r=r(\vec{\theta}, \vec{k} ; I)$. Moreover any additional power series in $\mathcal{F}_{I}$ with roots of order $k_{i}$ at the extremal $\alpha_{i}$ must have these same coefficients $c_{n}$ for $n \notin \mathcal{S}(\vec{\theta}, \vec{k}, \vec{N})$. 
We remark that (as will be apparent from the proof) the structure theorem in fact still holds if we allow the coefficients $a_{n}$ to lie in variable intervals $\left[B_{n}-A_{n}, B_{n}+A_{n}\right]$ containing zero (permitting versions for polynomials of bounded degree and so forth).

Notice that vanishing at $\vec{\theta}$ arguments with $\vec{k}$ multiplicities amounts really to solving $M(\vec{\theta}, \vec{k})$ equations (the real and imaginary parts of $f^{l}\left(r e^{2 \pi i \theta_{j}}\right), l=0, \ldots, k_{j}-1$, $j=1, \ldots, t)$. This simple observation is the main idea behind Theorem 1 . Moreover (assuming that the extremal series has at least $(M-1)$ non-dependent coefficients strictly inside $I$ ), for a choice of exponents $\vec{N}$, the structure theorem allows us to straightforwardly test whether we have picked the correct exponents. Since the values of the $a_{n}$ for $n$ not in $\mathcal{S}(\vec{\theta}, \vec{k}, \vec{N})$ are determined from the theorem, we can use $(M-1)$ of the $M$ equations to eliminate the $(M-1)$ unknown coefficients $a_{N_{i}}$ (along with any other remaining $a_{n}$ with $n \in \mathcal{S}(\vec{\theta}, \vec{k}, \vec{N})$ ) from the remaining equation, solve the result for $r$, and then, by checking whether this value of $r$ leads to $a_{n}$ in $I$ for all the missing $n \in \mathcal{S}(\vec{\theta}, \vec{k}, \vec{N})$, decide whether $r=r(\vec{\theta}, \vec{k} ; I)$. When $\theta$ is rational we have the added advantage that the form of such a power series allows us to reduce to an equivalent polynomial.

If zero is not an end point of $I$, then $r(\vec{\theta}, \vec{k} ; I)$ is essentially a continuous function of the arguments away from the real axis:

Lemma 2. Let $\vec{\phi}=\left(\phi, \theta_{2}, \ldots, \theta_{t}\right)$ with $\phi$ in $(0,1 / 2) \backslash\left\{\theta_{2}, \ldots, \theta_{t}\right\}$.

If $I=[U, V]$ with $U<0<V$, or if zero is an end point of $I$ and there is an extremal series for $r(\vec{\phi}, \vec{k} ; I)$ with $M(\vec{\phi}, \vec{k})$ non-zero coefficients $a_{n_{1}}, \ldots, a_{n_{M}}$ (allowed to include $a_{0}=1$ ) such that $\operatorname{det} B(\vec{\phi}, \vec{k}, \vec{N}) \neq 0, \vec{N}:=\left(n_{1}, \ldots, n_{M}\right)$, then

$$
\lim _{\theta_{1} \rightarrow \phi} r(\vec{\theta}, \vec{k} ; I)=r(\vec{\phi}, \vec{k} ; I) .
$$

For non-one-sided intervals this can be made quantitative (see Lemma 5 ) so that theoretically bounds on the value of $r(\theta, 2 ;[-1,1])$ for all $\theta$ could be obtained from computing a suitably fine mesh of rationals; in practice the bounds are too weak to be useable, although the picture we obtain suggests that the fluctuations are not particularly wild. The points $\phi$ in $\left\{0,1 / 2, \theta_{2}, \ldots, \theta_{t}\right\}$ are inevitably exceptional since, writing $\vec{\phi}=\left(\phi, \theta_{2}, \ldots, \theta_{t}\right)$ and $\vec{K}=\left(K, k_{2}, \ldots, k_{t}\right)$,

$$
\lim _{\theta_{1} \rightarrow \phi} r(\vec{\theta}, \vec{k} ; I) \geq r(\vec{\phi}, \vec{K} ; I)
$$

with $K=\mu k_{1}+k$, where $\mu=2$ if $\phi=0$ or $1 / 2$, and 1 otherwise, and $k=k_{j}$ if $\phi=\theta_{j}$ and 0 otherwise. Regarding $r(\theta, 2 ;[-1,1])$ we remark that $r(0,2 ;[-1,1])=$ $.6491378608 \ldots$, while the smallest fourth order real root

$$
r(0,4 ;[-1,1])=.7773295434 \ldots .
$$

This latter value (plotted as the point at zero in Figure 1) is probably the limit of $r(\theta, 2 ;[-1,1])$ as $\theta \rightarrow 0$. We remark that $r(0,4 ;[-1,1])$ corresponds to taking $\vec{N}=(4,9,19)$ in Theorem 1 (see [1]). In the case of one-sided intervals some additional restrictions are certainly needed to ensure continuity. For example if $\vec{\theta}:=(0, \theta), \vec{k}:=(1,1)$ and $I=[-1,0]$, then $r(\vec{\theta}, \vec{k} ; I)=(1 / 2)^{1 / q}$ for rational $\theta=p / q$ in $(0,1 / 2]$ (with extremal power series $1-\sum_{j=1}^{\infty} x^{j q}$ ), but is not defined for any irrational $\theta$ (the equations necessary for vanishing at $r>0$ and $r e^{2 \pi i \theta}$ clearly requiring $\cos (2 \pi n \theta)=1$ for any non-zero coefficients $a_{n}$ in the power series). However in the case of primary interest, $r(\theta, 2 ; I)$, it seems reasonable to expect 
continuity even for one-sided intervals such as $I=[0,1]$ or $[-1,0]$. It is certainly true that none of the points we computed could be discontinuities, since in all cases the extremal series had three coefficients strictly inside $I$ with $\operatorname{det} B\left(\theta, 2, \vec{N}_{0}\right) \neq 0$ (as in the statement of Theorem 1).

We now concentrate on computing the size, $r(\theta, 2 ; I)$, of the smallest double root on a given non-real ray, for rational arguments $\theta$.

\section{Computing the smallest Complex Double root FOR A GIVEN RATIONAL ARGUMENT}

Suppose now that $\theta>0$ is rational. Writing

$$
\theta:=p / Q, \quad(p, Q)=1,
$$

we define

$$
q:=\left\{\begin{array}{ll}
Q & \text { if } Q \text { is odd, } \\
Q / 2 & \text { if } Q \text { is even, }
\end{array} \quad \epsilon:= \begin{cases}1 & \text { if } Q \text { is odd } \\
-1 & \text { if } Q \text { is even. }\end{cases}\right.
$$

We shall assume that $\vec{N}=(N, M, R)$ satisfies the conditions of Theorem 1, where, without loss of generality $\sin (2 \pi N \theta) \neq 0$. Separating the coefficients into arithmetic progressions $\bmod q$, we observe that, for $n=l q+i$,

$$
\operatorname{sign}\left(\frac{\operatorname{det} B\left(\theta, 2, \vec{N}_{n}\right)}{\operatorname{det} B\left(\theta, 2, \vec{N}_{0}\right)}\right)=\operatorname{sign}\left(\alpha_{i} l-\beta_{i}\right) \epsilon^{l},
$$

where, writing

$$
\begin{aligned}
D_{n}(N, & M, R, \theta) \\
:= & (R-N)(n-M) \sin (2 \pi R \theta) \sin (2 \pi(N-M) \theta) \sin (2 \pi(N-n) \theta) \\
& +(M-N)(R-n) \sin (2 \pi M \theta) \sin (2 \pi(N-R) \theta) \sin (2 \pi(N-n) \theta) \\
& +(R-M)(N-n) \sin (2 \pi(N-M) \theta) \sin (2 \pi(N-R) \theta) \sin (2 \pi n \theta),
\end{aligned}
$$

we can explicitly write

$$
\beta_{i}:=-D_{i}(N, M, R, \theta) / D_{0}(N, M, R, \theta),
$$

and

$$
\begin{aligned}
\alpha_{i}:=q & \{(R-N) \sin (2 \pi R \theta) \sin (2 \pi(N-M) \theta) \sin (2 \pi(N-i) \theta) \\
& -(M-N) \sin (2 \pi M \theta) \sin (2 \pi(N-R) \theta) \sin (2 \pi(N-i) \theta) \\
& -(R-M) \sin (2 \pi(N-M) \theta) \sin (2 \pi(N-R) \theta) \sin (2 \pi i \theta)\} / D_{0}(N, M, R, \theta) .
\end{aligned}
$$

Notice that in the case of a $k$ th order root on a ray the linear polynomial $\left(\alpha_{i} l-\beta_{i}\right)$ would become a $(k-1)$ degree polynomial allowing for up to $(k-1)$ sign changes in each arithmetic progression of coefficients $a_{l q+i}$.

Hence, defining

$$
\lambda_{i}:=\left\{\begin{array}{ll}
\max \left\{0,\left[\beta_{i} / \alpha_{i}\right]+1\right\} & \text { if } \alpha_{i} \neq 0, \\
0 & \text { if } \alpha_{i}=0,
\end{array} \quad \kappa_{i}:= \begin{cases}\operatorname{sign}\left(\alpha_{i}\right) & \text { if } \alpha_{i} \neq 0, \\
-\operatorname{sign}\left(\beta_{i}\right) & \text { if } \alpha_{i}=0,\end{cases}\right.
$$

and writing $I:=[U, V]=[B-A, B+A]$, we readily see that the corresponding extremal power series must take the form

$$
F(x):=(1-U)+A \sum_{i=0}^{q-1} \kappa_{i} \frac{\left(1-2\left(\epsilon x^{q}\right)^{\lambda_{i}}\right)}{\left(1-\epsilon x^{q}\right)} x^{i}+\frac{B}{1-x}+E(x),
$$


where

$$
E(x):=\sum_{n \in \mathcal{S}(\theta, 2, \vec{N})}\left(a_{n}-B-A \kappa_{n}\right) x^{n} .
$$

Hence, eliminating the $a_{n}$ in $\mathcal{S}(\theta, 2, \vec{N})$, if $r e^{2 \pi i \theta}$ is to be a double root of $F$ then $r$ must be a root of

$$
P(x):=(1-U)-\sum_{i=0}^{q-1}\left(A \frac{P_{i}(x)}{\left(1-x^{q}\right)^{2}}+B \frac{Q_{i}(x)}{\left(1-\epsilon x^{q}\right)^{2}}\right) x^{i},
$$

where

$$
\begin{array}{r}
P_{i}(x):=\left|\beta_{i}\right|\left(1-x^{q}\right)\left(1-2 \max \left\{0, \operatorname{sign}\left(\alpha_{i} \beta_{i}\right)\right\} x^{\lambda_{i} q}\right) \\
+\left|\alpha_{i}\right|\left(2 \lambda_{i} x^{\lambda_{i} q}\left(1-x^{q}\right)-x^{q}\left(1-2 x^{\lambda_{i} q}\right)\right),
\end{array}
$$

and

$$
Q_{i}(x):=\beta_{i}\left(1-\epsilon x^{q}\right)-\alpha_{i} \epsilon x^{q} .
$$

We distinguish two cases: case 1 when $N, M$ and $R$ are all distinct modulo $q$ and the extremal power series is typically unique, and case 2 when $N \equiv R(\bmod q)$ and the extremal series will not be unique.

Case 1. In the usual situation the exponents $N, M$, and $R$ will be distinct modulo $q$ and, as long as $\mathcal{S}(\theta, 2, \vec{N})=\{N, M, R\}$, we can tell whether we have chosen the correct triple $N, M, R$ (and hence whether $r$ is the desired value) by testing whether the missing coefficients $a_{N}, a_{M}, a_{R}$ lie in $I$. Defining

$$
H(I, J, K):=-\frac{I \sin (2 \pi J \theta)}{r^{K}(I-K) \sin (2 \pi(J-K) \theta)}
$$

and

$$
L(I, J, K):=-\frac{\sum_{i=0}^{q-1} \kappa_{i} L_{i}(I) r^{i} \sin (2 \pi(J-i) \theta)}{r^{K}(I-K) \sin (2 \pi(J-K) \theta)\left(1-r^{q}\right)^{2}},
$$

where

$$
L_{i}(I):=\left(I-(I+q) r^{q}\right)\left(1-2 r^{\lambda_{i} q}\right)-\left(1-r^{q}\right)\left(i-2\left(i+\lambda_{i} q\right) r^{\lambda_{i} q}\right),
$$

and

$$
T(I, J, K)
$$

$$
:=\frac{\left(I \xi r+r^{3}\right) \sin (2 \pi(J+1) \theta)-\left(I \xi+2 r^{2}\right) \sin (2 \pi J \theta)+r \sin (2 \pi(J-1) \theta)}{\xi^{2} r^{K}(I-K) \sin (2 \pi(J-K) \theta)},
$$

where

$$
\xi:=\left(1-2 r \cos (2 \pi \theta)+r^{2}\right)
$$

we note that

$$
\begin{aligned}
a_{N} & =B+A \kappa_{N}+(1-U) H(R, M, N)+A L(R, M, N)+B T(R, M, N), \\
a_{M} & =B+A \kappa_{M}+(1-U) H(R, N, M)+A L(R, N, M)+B T(R, N, M), \\
a_{R} & =B+A \kappa_{R}+(1-U) H(N, M, R)+A L(N, M, R)+B T(N, M, R) .
\end{aligned}
$$

Even if $\mathcal{S}(\theta, 2, \vec{N})$ contains additional points we may still pick up the correct solution if there is an extremal series that can tolerate assigning $a_{n}=B+A \kappa_{n}$ for the remaining terms, although we knowingly encountered no such cases in our computations. Notice that if $\mathcal{S}(\theta, 2, \vec{N})=\{N, M, R\}$ then the extremal power series will be unique. 
Case 2. If two of the exponents are congruent $\bmod q$ then the parameters simplify quite drastically. Suppose that $N \equiv R \equiv s \bmod q$, where $1 \leq s \leq(q-1)$ (by Lemma 3 below we cannot have $s=0$ ), and $M \not \equiv s \bmod q$. Then

$$
\mathcal{S}(\theta, 2, \vec{N})=\{n: n \equiv s(\bmod q)\} \cup\{M\}
$$

with

$$
\alpha_{i}=-\frac{q}{M} \frac{\sin (2 \pi(s-i) \theta)}{\sin (2 \pi s \theta)}, \quad \beta_{i}=-\left(\frac{M-i}{M}\right) \frac{\sin (2 \pi(s-i) \theta)}{\sin (2 \pi s \theta)}
$$

and

$$
\lambda_{i}=\left[\frac{M+q-i}{q}\right], \quad \kappa_{i}=-\operatorname{sign}\left(\frac{\sin (2 \pi(s-i) \theta)}{\sin (2 \pi s \theta)}\right)
$$

where

$$
P(x)=(1-U)-\frac{1}{M} \sum_{i=0}^{q-1}\left|\frac{\sin (2 \pi(s-i) \theta)}{\sin (2 \pi s \theta)}\right|\left(A \frac{\tilde{P}_{i}(x)}{\left(1-x^{q}\right)^{2}}+B \kappa_{i} \frac{\tilde{Q}_{i}(x)}{\left(1-\epsilon x^{q}\right)^{2}}\right) x^{i},
$$

with

$$
\tilde{P}_{i}(x):=\left(M-(M+q) x^{q}\right)\left(1-2 x^{\lambda_{i} q}\right)-\left(1-x^{q}\right)\left(i-2\left(i+\lambda_{i} q\right) x^{\lambda_{i} q}\right)
$$

and

$$
\tilde{Q}_{i}(x):=(M-i)\left(1-\epsilon x^{q}\right)-q \epsilon x^{q} .
$$

Moreover, the following lemma tells us that for some equivalent power series the coefficients indexed by an arithmetic progression $\bmod q$ can be assumed to have a specific form.

Lemma 3. Suppose that $\theta=p / Q$ is rational as above. Then there is an extremal power series $f(x)=1+\sum_{i=1}^{\infty} a_{i} x^{i}$ in $\mathcal{F}_{I}$ with a double root at $\alpha=r(\theta, 2 ; I) e^{2 \pi i \theta}$ with the property that for each $i=1, \ldots,(q-1)$ there is a constant $C_{i}$ such that the coefficients of $f$ satisfy either

$$
\left(a_{l q+i}-B\right) \epsilon^{l}=C_{i} \quad \text { for all } l \geq 0
$$

or

$$
\left(a_{l q+i}-B\right) \epsilon^{l}= \begin{cases}C_{i} & \text { for } 0 \leq l<N_{i}, \\ C_{i}^{\prime} & \text { for } l=N_{i}, \\ \operatorname{sign}\left(C_{i}^{\prime}-C_{i}\right) A & \text { for } l>N_{i},\end{cases}
$$

for some constant $C_{i}^{\prime} \neq C_{i}$ and integer $N_{i} \geq 1$.

For $i=0$ the coefficients of any extremal series must satisfy

$$
\left(a_{l q}-B\right) \epsilon^{l}=-A \quad \text { for all } l \geq 1,
$$

or

$$
\left(a_{l q}-B\right) \epsilon^{l}=+A \quad \text { for all } l \geq 1,
$$

or

$$
\left(a_{l q}-B\right) \epsilon^{l}= \begin{cases}-A & \text { for } 1 \leq l<N_{0} \\ C_{0}^{\prime} & \text { for } l=N_{0} \\ +A & \text { for } l>N_{0}\end{cases}
$$

for some constant $C_{0}^{\prime}$ and integer $N_{0} \geq 1$. 
Hence we can assume that

(1)

$$
E(x)=\left(a_{M}-B-A \kappa_{M}\right) x^{M}+x^{s}\left(\frac{C_{1}\left(1-\left(\epsilon x^{q}\right)^{n}\right)}{\left(1-\epsilon x^{q}\right)}+C_{2}\left(\epsilon x^{q}\right)^{n} \pm \frac{A\left(\epsilon x^{q}\right)^{(n+1)}}{\left(1-\epsilon x^{q}\right)}\right)
$$

for some integer $n$, and $\left|C_{i}\right| \leq A$, regarding the possible case

$$
E(x)=\left(a_{M}-B-A \kappa_{M}\right) x^{M}+\frac{C x^{s}}{\left(1-\epsilon x^{q}\right)}
$$

as corresponding to $n=\infty$ (we encountered only one such case, corresponding to $I=[-1,1], \theta=1 / 4$, and $C=0)$. We can thus check whether we potentially have the correct $M$ and $s$ by checking whether the root $r$ of $P$ gives

$$
\begin{gathered}
a_{M}=B+A \kappa_{M}-\frac{(1-U) \sin (2 \pi s \theta)}{r^{M} \sin (2 \pi(s-M) \theta)}-A \sum_{i=0}^{q-1} \kappa_{i} \frac{\left(1-2 r^{\lambda_{i} q}\right)}{\left(1-r^{q}\right)} \frac{r^{i} \sin (2 \pi(s-i) \theta)}{r^{M} \sin (2 \pi(s-M) \theta)} \\
-B \frac{(\sin (2 \pi s \theta)-r \sin (2 \pi(s+1) \theta))}{\xi r^{M} \sin (2 \pi(s-M) \theta)}
\end{gathered}
$$

with $a_{M}$ in $I$, and in these few cases vary $n$ to see whether, writing

$$
\begin{aligned}
U^{ \pm}:= & (1-U)\left(1-r^{q}\right) \sin (2 \pi M \theta)-A \sum_{i=0}^{q-1} \kappa_{i}\left(1-2 r^{\lambda_{i} q}\right) r^{i} \sin (2 \pi(M-i) \theta) \\
& \mp A r^{s+(n+1) q} \sin (2 \pi(M-s) \theta) \\
& -B \frac{\left(1-r^{q}\right)}{\xi}(\sin (2 \pi M \theta)-r \sin (2 \pi(M+1) \theta)),
\end{aligned}
$$

and

$$
\begin{aligned}
V^{ \pm}:=( & 1-U) q r^{q} \sin (2 \pi M \theta)-A \sum_{i=0}^{q-1} \kappa_{i}\left(i-2\left(i+\lambda_{i} q\right) r^{\lambda_{i} q}\right) r^{i} \sin ((M-i) \theta) \\
& \mp A(s+(n+1) q) r^{s+(n+1) q} \sin (2 \pi(M-s) \theta) \\
& +B \frac{q r^{q}}{\xi}(\sin (2 \pi M \theta)-r \sin (2 \pi(M+1) \theta)) \\
& -B \frac{\left(1-r^{q}\right)}{\xi^{2}}\left(r \sin (2 \pi(M-1) \theta)-2 r^{2} \sin (2 \pi M \theta)+r^{3} \sin (2 \pi(M+1) \theta)\right),
\end{aligned}
$$

the missing coefficients

$$
C_{1}^{ \pm}:=\frac{\left((s+n q)-(s+(n+1) q) r^{q}\right) U^{ \pm}-\left(1-r^{q}\right) V^{ \pm}}{q r^{s} \sin (2 \pi(M-s) \theta)\left(r^{(n+1) q}+n-(n+1) r^{q}\right)}
$$

and

$$
C_{2}^{ \pm}:=\frac{\left(1-r^{n q}\right) V^{ \pm}-\left(s-(n q+s) r^{n q}\right) U^{ \pm}}{q r^{n q+s} \sin (2 \pi(M-s) \theta)\left(r^{(n+1) q}+n-(n+1) r^{q}\right)},
$$

satisfy $\left|C_{1}^{+}\right|,\left|C_{2}^{+}\right| \leq A$ or $\left|C_{1}^{-}\right|,\left|C_{2}^{-}\right| \leq A$. If $\left|C_{1}\right|$ and $\left|C_{2}\right|<A$ then the extremal power series will not be unique (the argument is as in the proof of Lemma 3 ). 
We computed the value of $r(\theta, 2 ;[-1,1])$ for all rationals $\theta$ in $(0,1 / 4]$ with $q \leq 50$ and $r(\theta, 2 ;[0,1])$ and $r(\theta, 2 ;[-1,0])$ for all $\theta$ in $(0,1 / 2)$ with $Q \leq 50$ (for the nonsymmetrical intervals, $B \neq 0$, there is no degree gain for the even denominators $Q=2 q)$. The computations were performed in Maple, using a supplementary real root finder (courtesy of Franck Beaucoup) to deal with the cases of high degree. The results are plotted in Figures 1,3 and 5, respectively (with the smallest fourth order roots rather than the smallest double roots plotted for the real points $\theta=0$ or $1 / 2$ ). For $I:=[-1,1]$ we encountered only 13 'case 2' rationals, 52 (out of 386) for $I=[0,1]$, and 78 for $[-1,0]$. The exponents $[M, N, R]$ for the 'case 1 ' rationals, or $[M, s, n q+s]$ with minimal successful $n$ for the 'case 2' rationals, are shown in Tables 1, 2 and 3 at the end of this paper (the 'case 2' distinguished by an asterisk). We also plot these three values against $\theta$ in Figures 2, 4 and 6 . It is noticeable that the smallest of the three exponents seems to resemble the exponent involved in computing the smallest single root on a ray (see [2]). As can be seen from Figures 2, 4 and 6, the initially daunting task of finding the correct three exponents $N, M, R$ for each of the rationals searched was made easier by the tendency of the exponents to take the same values as their neighbours. Cases of 'jumps' were dealt with by comparing the power series corresponding to nearby 'near misses' for the location of the first few differences. Notice also that the correct value of $r$ must be the largest root encountered as $N, M$ and $R$ are varied (the other roots correspond to extremal solutions where the size of some of the intervals are increased). Thus any choice of exponents yields a lower bound on $r(\theta, 2 ; I)$ (an observation that could perhaps be used to compute an absolute lower bound for $r(\theta, 2 ;[-1,1]))$.

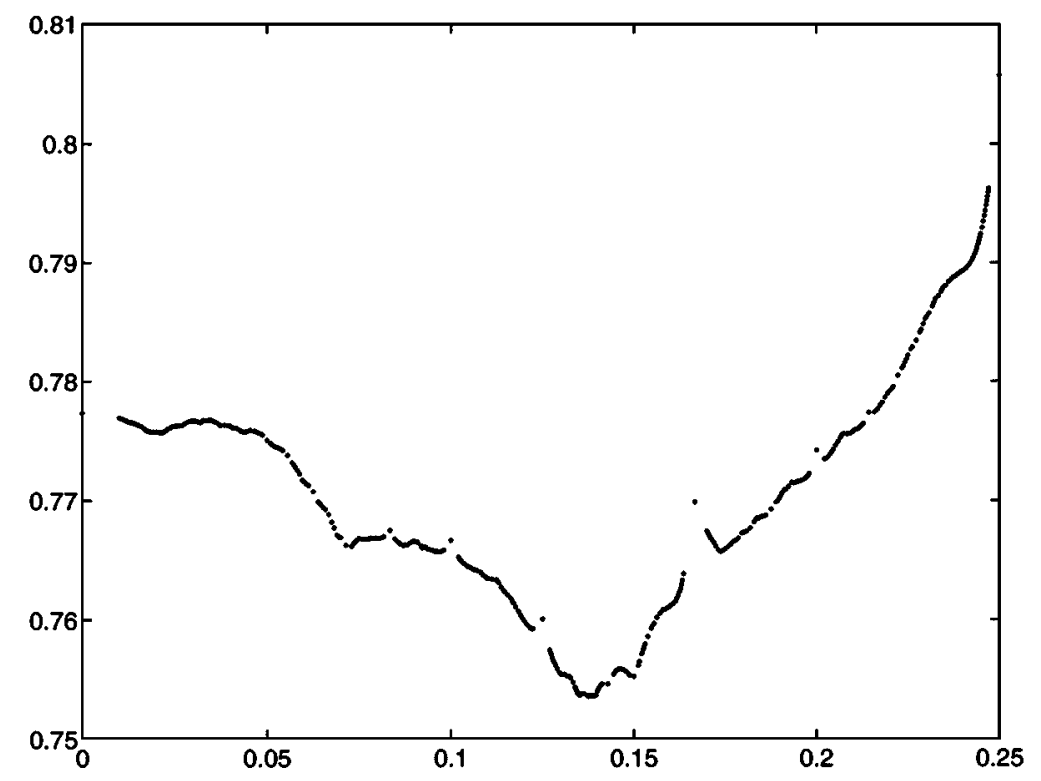

Figure 1. $r(\theta, 2 ; I)$ for $I=[-1,1]$, with $\theta$ in $(0,1 / 4]$. 


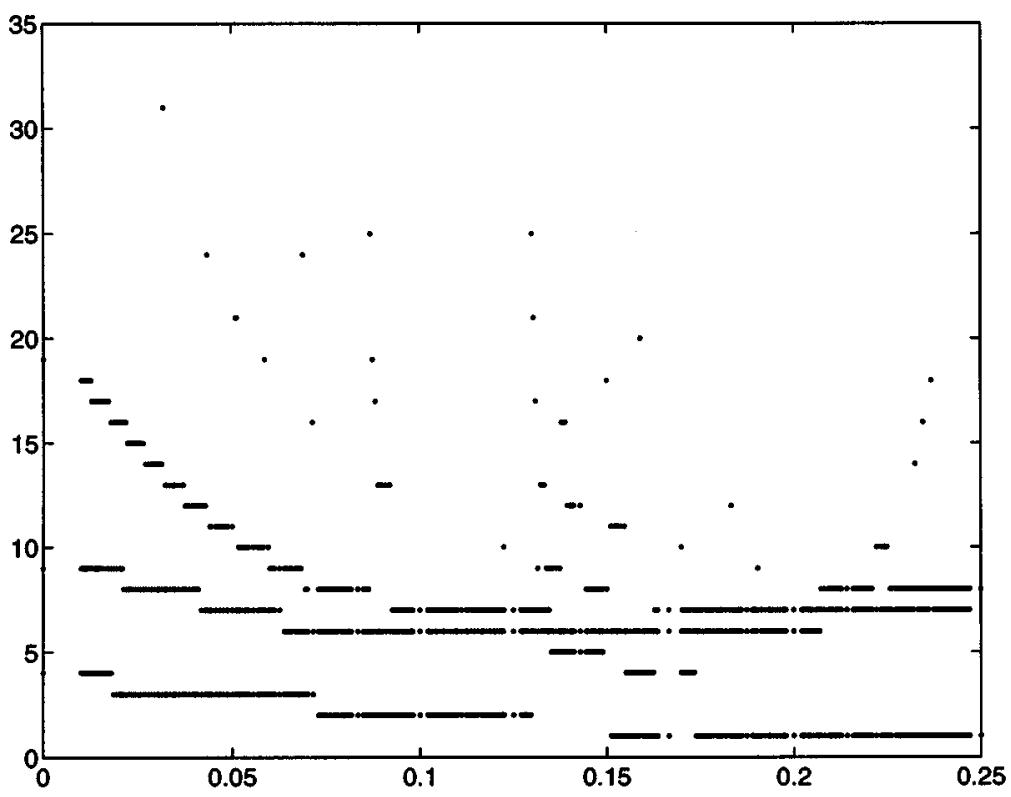

Figure 2. Successful values of $N, M, R$ for $I=[-1,1]$.

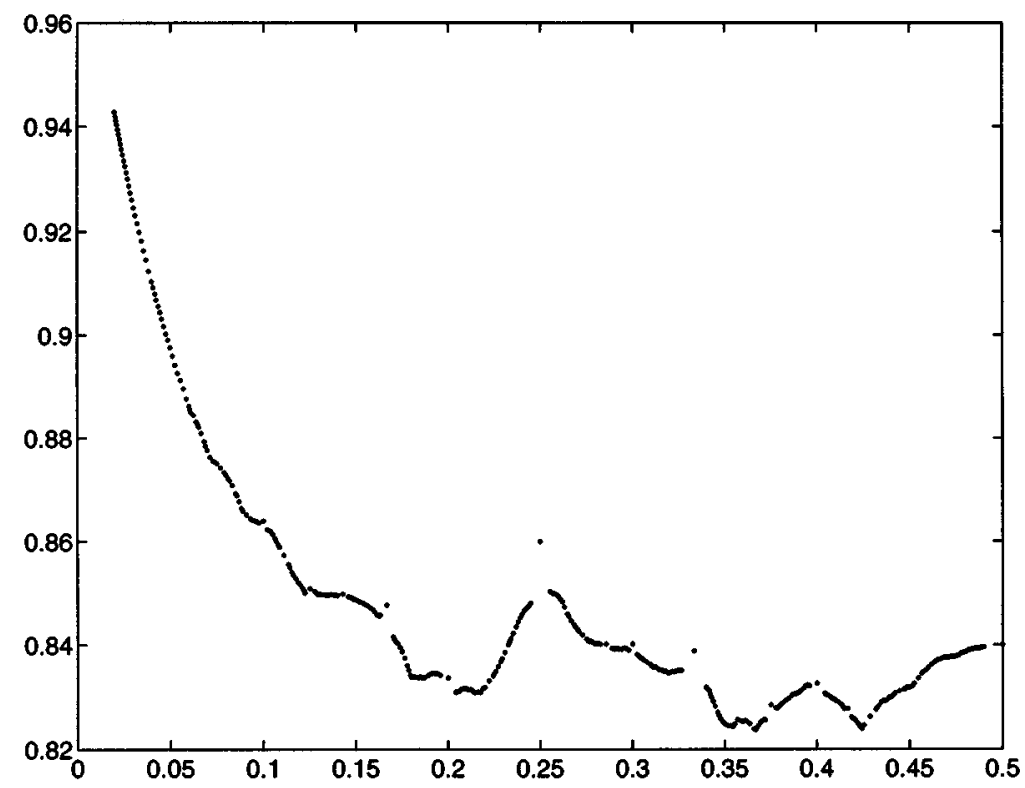

FIgURE 3. $r(\theta, 2 ; I)$ for $I=[0,1]$, with $\theta$ in $(0,1 / 2)$. 


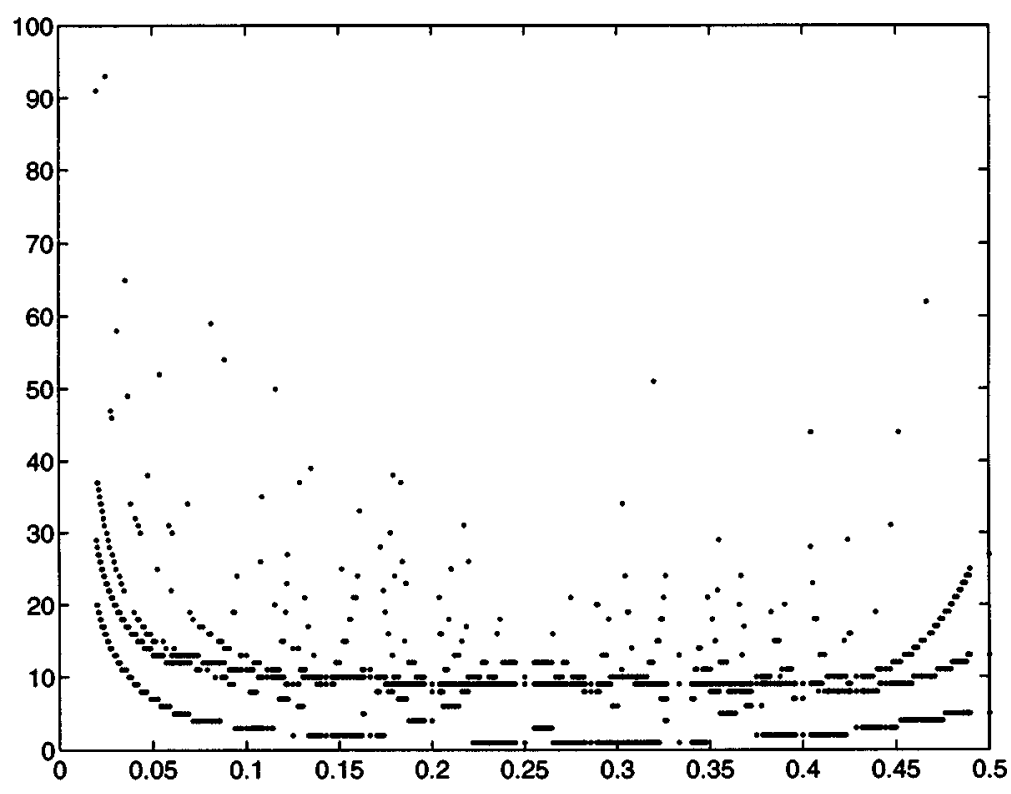

Figure 4. Successful values of $N, M, R$ for $I=[0,1]$.

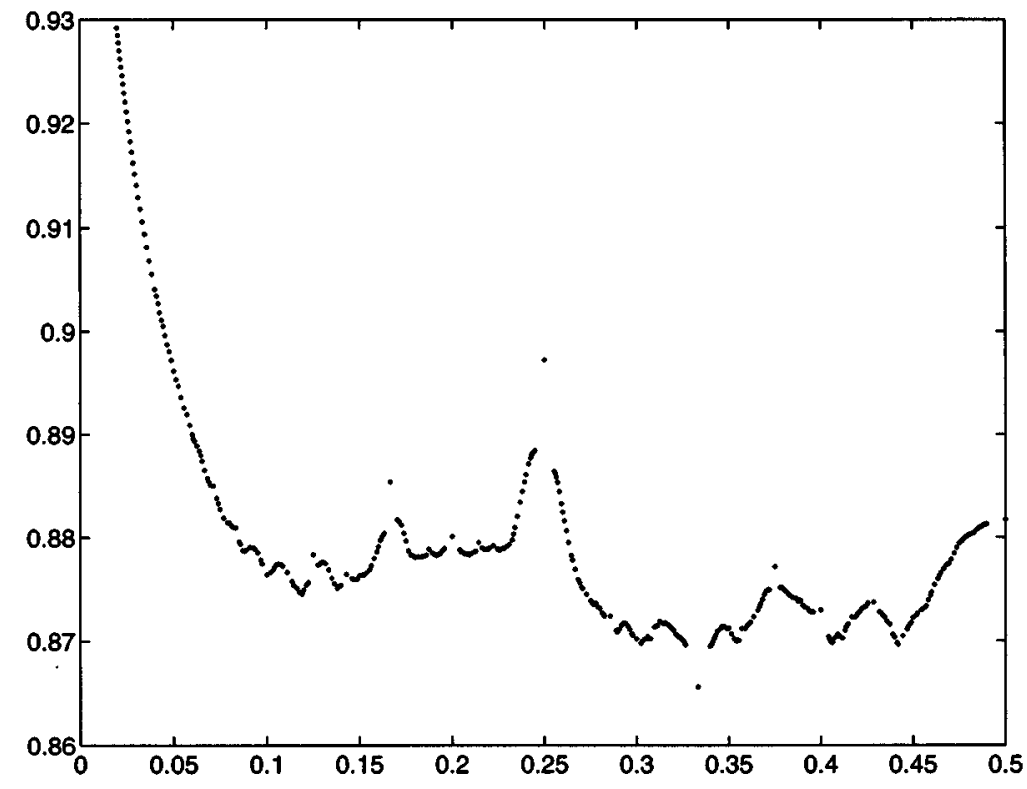

Figure 5. $r(\theta, 2 ; I)$ for $I=[-1,0]$, with $\theta$ in $(0,1 / 2)$. 


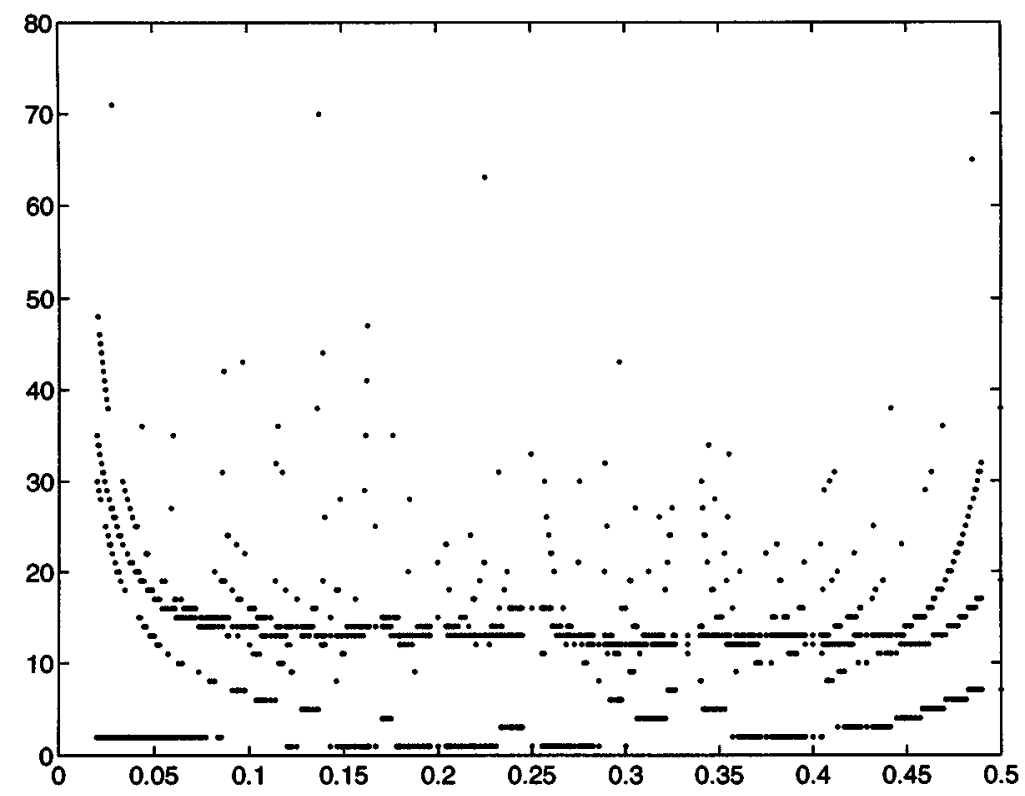

Figure 6. Successful values of $N, M, R$ for $I=[-1,0]$.

\section{Multiple Real roots}

For $k$ th order positive real roots we showed in [1] that the extremal power series has exactly $k$ sign changes and at most $(k-1)$ coefficients not equal to an end point (these occurring at the sign changes). That is, for $I=[B-A, B+A]$, the extremal series for $r(1 / 2, k ; I)$ takes the form $f(-x)$, where

$$
f(x)=1-\frac{B x}{1+x}-\frac{A x}{1-x}-\frac{2 A}{1-x} \sum_{i=1}^{k-1}(-1)^{i}\left(\beta_{i}+\left(1-\beta_{i}\right) x\right) x^{n_{i}}
$$

for some vector of exponents $\vec{N}=\left(n_{1}, \ldots, n_{k-1}\right)$ and coefficients $\beta_{i}$ in [0,1]. In [1] the value of $r(1 / 2, k ;[-1,1])=r(0, k ;[-1,1])$ is given for $k \leq 27$. In Tables 4 and 5 (at the end of this paper) we give $r(1 / 2, k ;[0,1])$ and $r(1 / 2, k ;[-1,0])$ for $k \leq 20$. When $I \subseteq[-1,1]$ we know, from bounds of Borwein, Erdélyi \& Kós [3], that

$$
r(1 / 2, k ; I) \geq 1-\frac{c}{k}
$$

for some absolute constant $c>0$. Conversely, in [1] we showed that

$$
r(1 / 2, k ;[-1,1]) \leq 1-\frac{1}{(k+1)},
$$

and more generally that $r(1 / 2, k ; I) \leq(k /(k+1))^{\min \{1,|U| / 9,|V| / 9\}}$. Similar bounds hold for one-sided intervals: 


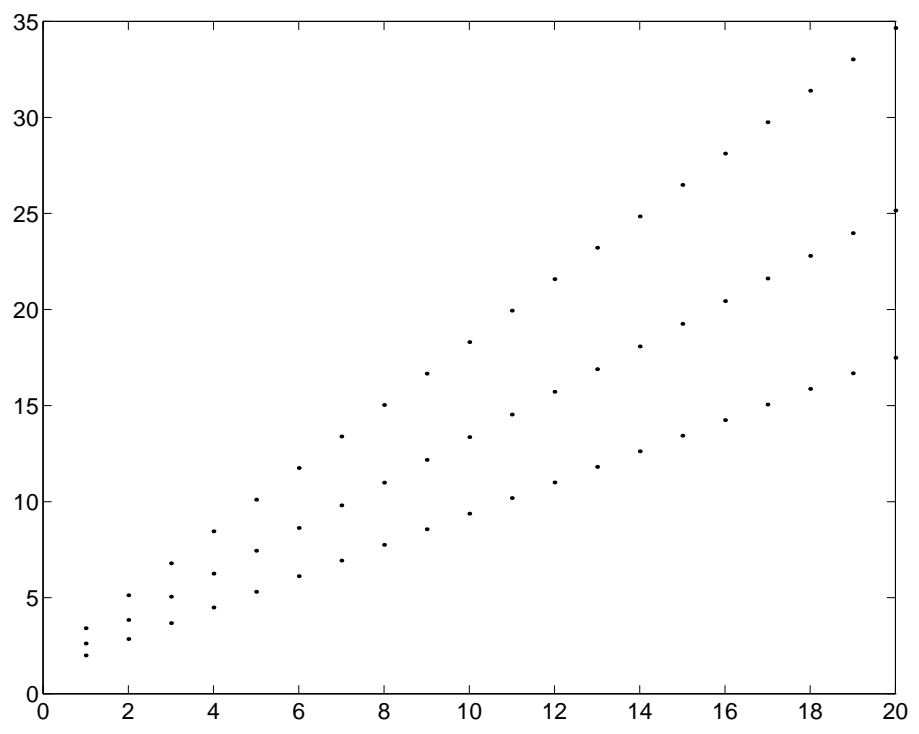

Figure 7. $(1-r(1 / 2, k ; I))^{-1}$ against $k$ for $I=[-1,1]$ (lower points), $[0,1]$ and $[-1,0]$ (upper points).

Proposition 1. Let $b_{i}$ denote the ith odd, square-free, positive integer. Then

$$
r(1 / 2, k ;[0, V]) \leq\left(1-\frac{1}{k+1}\right)^{1 / b_{s}}, \quad r(1 / 2, k ;[U, 0]) \leq\left(1-\frac{1}{k+1}\right)^{1 / 7 b_{s^{\prime}}},
$$

where $s:=\lceil 2 /|V|\rceil$ and $s^{\prime}:=\lceil 2 /|U|\rceil$.

Hence $(1-r(1 / 2, k ; I))^{-1}$ is of order precisely $k$ for $I=[-1,1],[0,1]$ and $[-1,0]$. Figure 7 contains a plot of $(1-r(1 / 2, k ; I))^{-1}$ against $k$ for these three intervals showing that the behaviour is not only bounded by linear functions but in fact approximates remarkably well to straight lines. The corresponding least squares lines are $1.51018740+1.18263768 k, 1.87642318+1.64050207 k$, and $1.23909318+$ $.81255949 k$ for $[0,1],[-1,0]$ and $[-1,1]$ respectively. Finally we observe that as the size of the coefficients of the power series increases the situation appears to become computationally simpler. In particular, for large enough $A$ the smallest $k$ th order real root $r(0, k ;[-A, A])$ can be determined precisely, the vector $\vec{N}$ of Theorem 1 reducing in that case to $(1, \ldots, k-1)$;

Proposition 2. For $A \geq\left(2^{1 / k}-1\right)^{-k}$,

$$
r(0, k ;[-A, A])=\frac{1}{A^{1 / k}+1} .
$$

It is tempting to speculate that for $A \geq\left(2^{1 / 2 k}-1\right)^{-2 k}$ the smallest $k$ th order root $r(\theta, k ;[-A, A])$ corresponds to taking $\vec{N}=(1, \ldots, 2 k-1)$ in Theorem 1 for all $\theta$ in $(0,1 / 2)$, and that perhaps $\left(A^{1 / 2 k}+1\right)^{-1} \leq r(\theta, k ;[-A, A]) \leq\left(A^{1 / k}+1\right)^{-\frac{1}{2}}$ (these being the values as $\theta \rightarrow 0$ and at $\theta=1 / 4$ respectively). This is true for $k=1$ (see [2]) and appears from our computations to be true for $k=2$ (see Figure 8). 


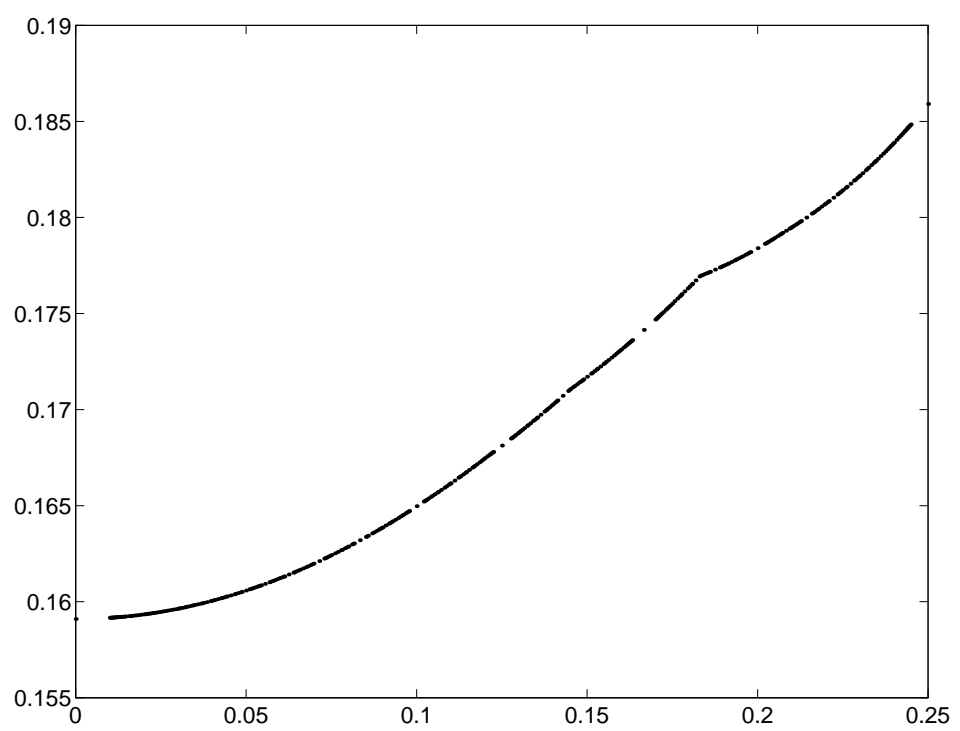

Figure 8. $r(\theta, 2 ; I)$ for $I=[-A, A], A=\left(2^{1 / 4}-1\right)^{-4}$.

\section{The PROOFS}

Proof of Lemma 1. Observe that the polynomial

$$
f(x ; n, \theta):=\left(x^{2 n}-2 \cos (2 \pi n \theta) x^{n}+1\right)^{\frac{1}{2} \delta(\theta)}
$$

has a root at $e^{2 \pi i \theta}$. Hence for a set of $N$ distinct primes $p_{i}$ with $M(\vec{\theta}, \vec{k})<p_{1}<$ $\cdots<p_{N}=p$ the polynomial

$$
F_{N}(x):=\frac{1}{N} \sum_{l=1}^{N} \prod_{j=1}^{t} f\left(x ; p_{l}, \theta_{j}\right)^{k_{j}}
$$

is a real polynomial of degree $M p$ with constant coefficient 1 and remaining coefficients of absolute value less than $2^{M} / N$, and a $k_{j}$ th order root at $e^{2 \pi i \theta_{j}}, j=1, \ldots, t$. Hence for $N \geq 2^{M+1} / A$ the polynomial $F_{N}\left(2^{1 / M p} x\right)$ is a polynomial with coefficients in $[-A, A]$ and a $k_{j}$ th order root at $2^{-1 / M p} e^{2 \pi i \theta_{j}}, j=1, \ldots, t$.

In the case of totally positive or totally negative intervals we similarly build polynomials

or

$$
F_{N}(x):=\frac{1}{N} \sum_{l=1}^{N} \prod_{j=1}^{t} f\left(x ; u_{l j} p_{l}, \theta_{j}\right)^{k_{j}}
$$

$$
F_{N}(x):=\frac{1}{N} \sum_{l=1}^{N}\left(\prod_{j=1}^{t} f\left(x ; u_{l j} p_{l}, \theta_{j}\right)^{k_{j}}\right)\left(2-\prod_{j=1}^{t} f\left(x ; u_{l j} p_{l}, \theta_{j}\right)^{k_{j}}\right)
$$

respectively, where the $u_{l j}$ are positive integers such that $\cos \left(2 \pi u_{l j} p_{l} \theta_{j}\right) \leq 0$ and the $p_{l}$ are primes greater than $2 M$, successively chosen so that $p_{l} \theta_{j} \not \equiv 0(\bmod 1)$ and $p_{l}>u_{s j} p_{s}$ for all $j$ and all $s<l$, and $N$ is taken suitably large.

For the proof of Theorem 1 we shall need the following simple lemma: 
Lemma 4. An extremal power series $f(x)=1+\sum_{i=1}^{\infty} a_{i} x^{i}$ in $\mathcal{F}_{I}$ (that is, a power series with a $k_{j}$ th order root at $\alpha_{j}=r(\vec{\theta}, \vec{k} ; I) e^{2 \pi i \theta_{j}}$ for $\left.j=1, \ldots, t\right)$ cannot have all its non-zero coefficients $a_{i}$ strictly inside $I$.

Proof. Suppose that $f(x)=1+\sum_{i=1}^{\infty} a_{i} x^{i}$ is an extremal polynomial with a $k_{j}$ th order root at $\alpha_{j}:=r e^{2 \pi i \theta_{j}}, r=r(\vec{\theta}, \vec{k} ; I)$, for $j=1, \ldots, t$, and all its non-zero $a_{i}$ strictly inside $I$. If $f(x)$ happened to be a polynomial

$$
f(x)=1+\sum_{i=1}^{J} a_{i} x^{i}, \quad \frac{U}{(1+\delta)}<a_{i}<\frac{V}{(1+\delta)},
$$

for some $\delta>0$, then the result would be almost trivial as the series $f\left((1+\delta)^{1 / J} x\right)$ would still be in $\mathcal{F}_{I}$ but would have smaller roots $(1+\delta)^{-1 / J} \alpha_{j}$, contradicting the minimality of $r$. Hence it is enough to show that we can replace the power series by a polynomial.

Writing

$$
f_{N}(x):=1+\sum_{n=1}^{N} a_{n} x^{n}, \quad h_{N}(x):=\sum_{n=N+1}^{\infty} a_{n} x^{n},
$$

we observe that for suitably large $N$ we can make the derivatives of the remainders $h_{N}$ arbitrarily small:

$$
\left|\frac{\alpha_{j}^{l} h_{N}^{l}\left(\alpha_{j}\right)}{l !}\right| \leq \max \{|U|,|V|\} \frac{N^{l} r^{N}}{(1-r)^{l+1}} .
$$

Let $V$ denote the real vector space generated by the $M(\vec{\theta}, \vec{k})$ dimensional vectors $\vec{v}_{n}:=\left(\cdots,\left(\begin{array}{c}n \\ l\end{array}\right) \cos \left(2 \pi n \theta_{j}\right),\left(\begin{array}{c}n \\ l\end{array}\right) \sin \left(2 \pi n \theta_{j}\right), \cdots\right), l=0, \ldots, k_{j}-1, j=$ $1, \ldots, t$, for the $n$ such that $a_{n} \neq 0$. Taking an $\vec{N}=\left(n_{1}, \ldots, n_{R}\right)$ such that the $\vec{v}_{n_{i}}$ form a basis for $V$, we can construct a real polynomial

$$
g_{N}(x):=\sum_{i=1}^{R} \gamma_{i}(N)\left(\frac{x}{r}\right)^{n_{i}}
$$

such that $\alpha_{j}^{l} g^{l}\left(\alpha_{j}\right) / l !=\alpha_{j}^{l} h_{N}^{l}\left(\alpha_{j}\right) / l !, l=0, \ldots, k_{j}-1, j=1, \ldots, t$. Moreover, by Cramer's rule, writing $\gamma_{i}(N)=\operatorname{det} M_{j}\left(\vec{u}_{N}\right) / \operatorname{det} M$, where $M$ is a matrix formed from $R$ linearly independent rows of $\left(\vec{v}_{n_{1}}^{T}, \ldots, \vec{v}_{n_{R}}^{T}\right), \vec{u}_{N}$ is the vector consisting of the $R$ real or imaginary parts of the derivatives $\alpha_{j}^{l} h_{N}^{l}\left(\alpha_{j}\right) / l$ ! corresponding to the $R$ chosen rows, and $M_{i}(\vec{u})$ denotes the matrix obtained by replacing the $i$ th column of $M$ by $\vec{u}$, we clearly have $\left|\gamma_{i}(N)\right| \leq C \max _{i}\left|u_{i}\right|$. Thus for a suitably large $N$ we will have $a_{n_{i}}+\gamma_{i}\left(\vec{u}_{N}\right)$ strictly inside $I$, and $\tilde{f}(x):=f_{N}(x)+g_{N}(x)$ will be a polynomial with a $k_{j}$ th order root at $\alpha_{j}$ and all its non-zero coefficients strictly inside $I$.

Proof of Theorem 1. Let $\vec{N}=\left(N_{1}, \ldots, N_{(M-1)}\right)$ be a vector of $(M(\vec{\theta}, \vec{k})-1)$ positive integers. Observe that if $\operatorname{det} B\left(\vec{\theta}, \vec{k}, \vec{N}_{n}\right) \neq 0$ then, writing

$$
\vec{N}(i):=\left(N_{1}, \ldots, N_{i-1}, 0, N_{i+1}, \ldots N_{(M-1)}\right),
$$

by Cramer's rule,

$$
G(x ; \vec{\theta}, \vec{k}, \vec{N}, r):=1-\frac{\operatorname{det} B\left(\vec{\theta}, \vec{k}, \vec{N}_{0}\right)}{\operatorname{det} B\left(\vec{\theta}, \vec{k}, \vec{N}_{n}\right)}\left(\frac{x}{r}\right)^{n}-\sum_{i=1}^{M-1} \frac{\operatorname{det} B\left(\vec{\theta}, \vec{k}, \vec{N}(i)_{n}\right)}{\operatorname{det} B\left(\vec{\theta}, \vec{k}, \vec{N}_{n}\right)}\left(\frac{x}{r}\right)^{N_{i}}
$$

is a real polynomial with $G^{l}\left(r e^{2 \pi i \theta_{j}}\right)=0$ for $l=0, \ldots, k_{j}-1, j=1, \ldots, t$. 
Suppose then that $F(x)=1+\sum_{i=1}^{\infty} a_{i} x^{i}$ is an extremal power series in $\mathcal{F}_{I}$ with a $k_{j}$ th order root at $\alpha_{j}:=r(\vec{\theta}, \vec{k} ; I) e^{2 \pi i \theta_{j}}, j=1, \ldots, t$, and $U<a_{N_{i}}<V$ for $i=1, \ldots,(M-1)$. Suppose that $n$ is such that $\operatorname{det} B\left(\vec{\theta}, \vec{k}, \vec{N}_{n}\right) \neq 0$ and either $\operatorname{det} B\left(\vec{\theta}, \vec{k}, \vec{N}_{0}\right)=0$ or

$$
a_{n} \neq B-\operatorname{sign}\left(\frac{\operatorname{det} B\left(\vec{\theta}, \vec{k}, \vec{N}_{0}\right)}{\operatorname{det} B\left(\vec{\theta}, \vec{k}, \vec{N}_{n}\right)}\right) A .
$$

Then for a sufficiently small $\delta>0$ the power series

$$
\tilde{F}(x):=(1-\delta) F(x)+\delta G(x ; \vec{\theta}, \vec{k}, \vec{N}, r(\vec{\theta}, \vec{k} ; I))=1+\sum_{i=1}^{\infty} \tilde{a}_{i} x^{i}
$$

will be a power series with roots of order $k_{j}$ at the $\alpha_{j}$ with all its non-zero coefficients $\tilde{a}_{i}$ strictly inside $I$, contradicting Lemma 4 . Hence either $\operatorname{det} B\left(\vec{\theta}, \vec{k}, \vec{N}_{n}\right)$ is identically zero, or $\operatorname{det} B\left(\vec{\theta}, \vec{k}, \vec{N}_{0}\right) \neq 0$ and

$$
a_{n}=B-\operatorname{sign}\left(\frac{\operatorname{det} B\left(\vec{\theta}, \vec{k}, \vec{N}_{0}\right)}{\operatorname{det} B\left(\vec{\theta}, \vec{k}, \vec{N}_{n}\right)}\right) A, \quad \text { for all } n \notin \mathcal{S}(\vec{\theta}, \vec{k}, \vec{N}) .
$$

Suppose that $f(x)=1+\sum_{i=1}^{\infty} a_{i} x^{i}$ has roots of order $k_{j}$ at points $\beta_{j}:=r e^{2 \pi i \theta_{j}}$, $j=1, \ldots, t$. Then the conditions $f^{l}\left(\beta_{j}\right)=0$, for $l=0, \ldots, k_{j}-1, j=1, \ldots, t$, are equivalent to the $M=M(\vec{\theta}, \vec{k})$ equations

$$
\sum_{n=0}^{\infty} n^{l} a_{n} r^{n} \cos \left(2 \pi n \theta_{j}\right)=0, \quad \sum_{n=0}^{\infty} n^{l} a_{n} r^{n} \sin \left(2 \pi n \theta_{j}\right)=0
$$

(the second equation disappearing for real $\left.\beta_{j}\right)$. When $\operatorname{det} B\left(\vec{\theta}, \vec{k}, \vec{N}_{0}\right) \neq 0$, eliminating $a_{N_{i}}, i=1, \ldots,(M-1)$, from these equations readily gives that $r$ satisfies

$$
1+\sum_{n \notin \mathcal{S}(\vec{\theta}, \vec{k}, \vec{N})} a_{n} \frac{\operatorname{det} B\left(\vec{\theta}, \vec{k}, \vec{N}_{n}\right)}{\operatorname{det} B\left(\vec{\theta}, \vec{k}, \vec{N}_{0}\right)} r^{n}=0
$$

Set

$$
S^{+}:=\left\{n: \frac{\operatorname{det} B\left(\vec{\theta}, \vec{k}, \vec{N}_{0}\right)}{\operatorname{det} B\left(\vec{\theta}, \vec{k}, \vec{N}_{n}\right)}>0\right\}, \quad S^{-}:=\left\{n: \frac{\operatorname{det} B\left(\vec{\theta}, \vec{k}, \vec{N}_{0}\right)}{\operatorname{det} B\left(\vec{\theta}, \vec{k}, \vec{N}_{n}\right)}<0\right\} .
$$

Now if

$$
a_{n}=B-\operatorname{sign}\left(\frac{\operatorname{det} B\left(\vec{\theta}, \vec{k}, \vec{N}_{0}\right)}{\operatorname{det} B\left(\vec{\theta}, \vec{k}, \vec{N}_{n}\right)}\right) A, \quad \text { for all } n \notin \mathcal{S}(\vec{\theta}, \vec{k}, \vec{N}),
$$

then $r$ is a root of the power series

$$
P(x)=1-|U| \sum_{n \in S^{+}}\left|\frac{\operatorname{det} B\left(\vec{\theta}, \vec{k}, \vec{N}_{n}\right)}{\operatorname{det} B\left(\vec{\theta}, \vec{k}, \vec{N}_{0}\right)}\right| x^{n}-|V| \sum_{n \in S^{-}}\left|\frac{\operatorname{det} B\left(\vec{\theta}, \vec{k}, \vec{N}_{n}\right)}{\operatorname{det} B\left(\vec{\theta}, \vec{k}, \vec{N}_{0}\right)}\right| x^{n},
$$

and $r=r(\vec{\theta}, \vec{k} ; I)$ since no smaller value of $r$ can be achieved with a different assignment of coefficients $\tilde{a}_{n} \in[U, V]$ for the $n \notin \mathcal{S}(\vec{\theta}, \vec{k}, \vec{N})$ (by Descartes' rule of signs $P(x)$ has at most one root $r>0$, hence $P(x) \geq 0$ on [0,r], while any different configuration of coefficients $\tilde{a}_{i}$ corresponding to other roots $\tilde{r} e^{2 \pi i \theta_{j}}$ would lead to a $\tilde{P}(x)>P(x)$ and hence a root $\tilde{r}>r)$. 
Proof of Lemma 2. For a given $\phi$ we write

$$
\vec{\phi}=\left(\phi, \theta_{2}, \ldots, \theta_{t}\right) \text { and } \vec{K}=\left(K, k_{2}, \ldots, k_{t}\right),
$$

where $\theta_{j}$ and $k_{j}$ are omitted if $\phi=\theta_{j}$, and $K=\mu k_{1}+k$, where $\mu=2$ if $\phi=0$ or $1 / 2$ and $\mu=1$ otherwise, and $k=k_{j}$ if $\phi=\theta_{j}$ and $k=0$ otherwise. Compactness readily gives

$$
\liminf _{\theta_{1} \rightarrow \phi} r(\vec{\theta}, \vec{k} ; I) \geq r(\vec{\phi}, \vec{K} ; I) .
$$

Hence it is enough to show that, for $\phi \in(0,1 / 2) \backslash\left\{\theta_{2}, \ldots, \theta_{t}\right\}$,

$$
\limsup _{\theta_{1} \rightarrow \phi} r(\vec{\theta}, \vec{k} ; I) \leq r(\vec{\phi}, \vec{k} ; I) .
$$

We in fact show the following quantitative version when zero is not an end point of $I$ :

Lemma 5. Suppose $I=[U, V]$ with $U<0<V$. For $\phi$ in $(0,1 / 2) \backslash\left\{\theta_{2}, \ldots, \theta_{t}\right\}$,

$$
r(\vec{\theta}, \vec{k} ; I) \leq\left(1+\delta_{1}\left|\theta_{1}-\phi\right|\right) r(\vec{\phi}, \vec{k} ; I)
$$

and for $\phi$ in $\left\{0,1 / 2, \theta_{2}, \ldots, \theta_{t}\right\}$, with $0<2 \pi\left|\theta_{i}-\phi\right| \leq 1$,

$$
r(\vec{\phi}, \vec{K} ; I) \leq\left(1+\delta_{2}\left|\theta_{1}-\phi\right|\right) r(\vec{\theta}, \vec{k} ; I),
$$

where

$$
\delta_{1}:=C(\vec{\theta}, \vec{k}, r(\vec{\phi}, \vec{k} ; I)) \max \left\{\left|\frac{U}{V}\right|,\left|\frac{V}{U}\right|\right\}
$$

with

$$
\begin{aligned}
C(\vec{\theta}, \vec{k}, r):= & \frac{2 \pi k_{1}(M ! \sqrt{M})^{M}}{r^{M}(1-r)^{k_{1}+1}} \prod_{\substack{i=1 \\
\delta\left(\theta_{i}\right)=2}}^{t} \frac{2^{k_{i}}}{\left|2 \sin \left(2 \pi \theta_{i}\right)\right|^{k_{i}^{2}}} \\
& \times \prod_{1 \leq i<j \leq t}\left|2 \sin \pi\left(\theta_{i} \pm \theta_{j}\right)\right|^{-\frac{1}{2} \delta\left(\theta_{i}\right) \delta\left(\theta_{j}\right) k_{i} k_{j}},
\end{aligned}
$$

and

$$
\delta_{2}:=C(\vec{\phi}, \vec{K}, r(\vec{\theta}, \vec{k} ; I)) \max \left\{\left|\frac{U}{V}\right|,\left|\frac{V}{U}\right|\right\} \frac{(K-1)^{\frac{1}{2}(K-1)}}{2^{\frac{1}{2}(\mu-1) k_{1}\left(k_{1}-1\right)}} \prod_{j=0}^{k_{1}-1} \frac{(K-1-\mu j) !}{j !} .
$$

Proof. Let $f(x)=1+\sum_{n=1}^{\infty} a_{n} x^{n}$ be a power series in $\mathcal{F}_{I}$ with roots of order $k_{1}$ at $\beta_{1}:=r e^{2 \pi i \phi}$ and of order $k_{j}$ at $\alpha_{j}:=r e^{2 \pi i \theta_{j}}, j=2, \ldots, t$, where $r:=r(\vec{\phi}, \vec{k} ; I)$. Then, with $\alpha_{1}:=r e^{2 \pi i \theta_{1}}$ and $W:=\max \{|U|,|V|\}$,

$$
\begin{aligned}
\left|\alpha_{1}^{l} \frac{f^{l}\left(\alpha_{1}\right)}{l !}\right| & =\frac{1}{l !}\left|\alpha_{1}^{l} f^{l}\left(\alpha_{1}\right)-\beta_{1}^{l} f^{l}\left(\beta_{1}\right)\right| \\
& \leq \frac{W}{l !} \sum_{n=1}^{\infty} n^{l} r^{n}\left|e^{2 \pi i n\left(\theta_{1}-\phi\right)}-1\right|=\frac{2 W}{l !} \sum_{n=1}^{\infty} n^{l} r^{n}\left|\sin \pi n\left(\theta_{1}-\phi\right)\right| \\
& \leq \frac{2 W \pi}{l !}\left|\theta_{1}-\phi\right| \sum_{n=1}^{\infty} n^{l+1} r^{n} \leq 2 W \pi\left|\theta_{1}-\phi\right|(l+1)(1-r)^{-(l+2)},
\end{aligned}
$$

with $\alpha_{i}^{l} f^{l}\left(\alpha_{i}\right) / l !=0, l=0, \ldots, k_{j}-1, j=2, \ldots, t$. 
Observe that for the vector $\vec{N}:=(1,2, \ldots, M)$ the determinant of $B(\vec{\theta}, \vec{k}, \vec{N})$ is non-zero. In fact, replacing the trigonometric functions by exponentials to give a Vandermonde determinant, we can evaluate $|\operatorname{det} B(\vec{\theta}, \vec{k}, \vec{N})|$ exactly:

$$
\left(\prod_{\substack{j=1 \\ \delta\left(\theta_{j}\right)=2}}^{t} 2^{-k_{j}}\left|2 \sin \left(2 \pi \theta_{j}\right)\right|^{k_{j}^{2}}\right)\left(\prod_{1 \leq l<j \leq t}\left|2 \sin \pi\left(\theta_{j} \pm \theta_{l}\right)\right|^{\frac{1}{2} \delta\left(\theta_{j}\right) \delta\left(\theta_{l}\right) k_{j} k_{l}}\right) .
$$

Hence, writing $\vec{u}$ for the $M$ dimensional vector formed from the real and imaginary parts of $\alpha_{j}^{l} f^{l}\left(\alpha_{j}\right) / l !, l=0, \ldots, k_{j}-1, j=1, \ldots, t$, and $B(i, \vec{u})$ for the matrix obtained by replacing the $i$ th column of $B$ by $\vec{u}$, by Cramer's rule there is a real polynomial

$$
g(x ; \vec{u}, r):=\sum_{i=1}^{M} \gamma_{i}(\vec{u})\left(\frac{x}{r}\right)^{i}, \quad \gamma_{i}(\vec{u}):=\frac{\operatorname{det} B(\vec{\theta}, \vec{k}, \vec{N})(i, \vec{u})}{\operatorname{det} B(\vec{\theta}, \vec{k}, \vec{N})},
$$

such that

$$
\alpha_{j}^{l} \frac{g^{l}\left(\alpha_{j}\right)}{l !}=\alpha_{j}^{l} \frac{f^{l}\left(\alpha_{j}\right)}{l !}, \quad l=0, \ldots, k_{j}, j=1, \ldots, t,
$$

with, by Hadamard's inequality,

$$
\begin{aligned}
\left|\frac{\gamma_{i}(\vec{u})}{r^{i}}\right| & \leq \frac{\max _{i}\left|u_{i}\right|}{r^{M}} \frac{(M ! \sqrt{M})^{M}}{|\operatorname{det} B(\vec{\theta}, \vec{k}, \vec{N})|} \\
& \leq \frac{2 W \pi\left|\phi-\theta_{1}\right| k_{1}}{r^{M}(1-r)^{k_{1}+1}} \frac{(M ! \sqrt{M})^{M}}{|\operatorname{det} B(\vec{\theta}, \vec{k}, \vec{N})|}=: \lambda \min \{|U|,|V|\} .
\end{aligned}
$$

Hence

$$
\tilde{f}(x)=f(x)-g(x ; \vec{u}, r)
$$

is a power series with roots of order $k_{j}$ at the $\alpha_{j}, j=1, \ldots, t$, and coefficients $\tilde{a}_{n}$ in $(1+\lambda) I$. Hence $\tilde{f}(x /(1+\lambda))$ is a power series in $\mathcal{F}_{I}$ with roots of order $k_{j}$ at $r(1+\lambda) e^{2 \pi i \theta_{j}}, j=1, \ldots, t$.

Suppose that $f(x)=\sum_{n=0}^{\infty} a_{n} x^{n}, a_{0}=1$, has roots of order $k_{1}, \ldots, k_{t}$ at $\alpha_{j}=$ $r e^{2 \pi i \theta_{j}}, j=1, \ldots, t, r:=r(\vec{\theta}, \vec{k} ; I)$. When $\phi \neq 0$ or $1 / 2$ let $M_{1}$ denote the $\left(k_{1}+k\right) \times\left(k_{1}+k\right)$ matrix whose first $k$ rows consist of ones along the diagonal and zeros elsewhere and whose final $k_{1}$ rows consist of the first $\left(k_{1}+k\right)$ Taylor coefficients of $x^{i} e^{x}, i=0, \ldots k_{1}-1$. When $\phi=0$ or $1 / 2$ set $M_{2}$ and $M_{3}$ to be the $\left(k_{1}+l\right) \times\left(k_{1}+l\right)$ matrices, $l=[(k+1) / 2]$ and $[k / 2]$ respectively, whose first $l$ rows consist of ones along the diagonal and zeros elsewhere and whose remaining $k_{1}$ rows consist of the even, respectively odd, indexed Taylor coefficients of $x^{i} \sin x$ or $x^{i} \cos x$ as appropriate, $i=0, \ldots, k_{1}-1$. Observe (writing as a Vandermonde determinant, using Hadamard's inequality and crude bounding) that the inverses of these matrices certainly have entries of absolute value less than

$$
\nu:=\frac{(K-1)^{\frac{1}{2}(K-1)}}{2^{\frac{1}{2}(\mu-1) k_{1}\left(k_{1}-1\right)}} \prod_{j=0}^{k_{1}-1} \frac{(K-1-\mu j) !}{j !} .
$$

Setting $u:=\left(\theta_{1}-\phi\right), 0<2 \pi|u| \leq 1$, and taking linear combinations of the equations $\sum_{n=0}^{\infty} a_{n} r^{n}(2 \pi n u)^{l} \cos (2 \pi n \phi+2 \pi n u)=0, \quad \sum_{n=0}^{\infty} a_{n} r^{n}(2 \pi n u)^{l} \sin (2 \pi n \phi+2 \pi n u)=0$, 
for $l=0, \ldots,\left(k_{1}-1\right)$, and

$$
\sum_{n=0}^{\infty} a_{n} r^{n}(2 \pi n u)^{l} \cos (2 \pi n \phi)=0, \quad \sum_{n=0}^{\infty} a_{n} r^{n}(2 \pi n u)^{l} \cos (2 \pi n \phi)=0,
$$

for $l=0, \ldots, k-1$, we readily see that the derivatives at $r e^{2 \pi i \phi}$

$$
\sum_{n=0}^{\infty} a_{n} r^{n} n^{l} \cos (2 \pi n \phi), \quad \sum_{n=0}^{\infty} a_{n} r^{n} n^{l} \sin (2 \pi n \phi), \quad l=0, \ldots, K-1
$$

have absolute values

$$
\leq(2 \pi u)(K \nu) W \sum_{n=1}^{\infty} \frac{n^{K}}{K !} r^{n} \leq \frac{2 \pi W K \nu u}{(1-r)^{(K+1)}} .
$$

Thus, writing $\tilde{M}=M(\vec{\phi}, \vec{K}), \tilde{N}=(1,2, \ldots, \tilde{M})$, we get a polynomial

$$
g(x)=\sum_{i=1}^{\tilde{M}} \gamma_{i}\left(\frac{x}{r}\right)^{i}, \quad\left|\gamma_{i}\right| \leq \frac{2 \pi W K \nu u}{r^{\tilde{M}}(1-r)^{K+1}} \frac{(\tilde{M} ! \sqrt{\tilde{M}})^{\tilde{M}}}{\operatorname{det} B(\vec{\phi}, \vec{K}, \overrightarrow{\tilde{N}})}
$$

such that $\tilde{f}(x)=f(x)-g(x)$ has roots of order $k_{j}$ at $\alpha_{j}, j=2, \ldots, t$, and a root of order $K$ at $r e^{2 \pi i \phi}$. The result follows as before.

When zero is an end point of the interval $I$, and the extremal series contains $M(\vec{\theta}, \vec{k})$ non-zero coefficients $a_{n_{i}}$ with $\operatorname{det}(\vec{\phi}, \vec{k}, \vec{N}) \neq 0$, one can still obtain an analogue of the first bound (although now dependent upon the $n_{i}$ ) and hence deduce continuity at that point. For $\theta_{1}$ close enough to $\phi$ that $\operatorname{det}(\vec{\theta}, \vec{k}, \vec{N})$ is bounded away from zero, one similarly constructs a polynomial, $g(x):=\sum_{i=1}^{M} \gamma_{i}(x / r)^{n_{i}}$ such that $g^{l}\left(\alpha_{j}\right) / l !=f^{l}\left(\alpha_{j}\right) / l$ ! for $\alpha_{j}:=r(\vec{\phi}, \vec{k} ; I) e^{2 \pi i \theta_{j}}, l=0, \ldots, k_{j}-1, j=1, \ldots, t$, with $\gamma_{i}:=\operatorname{det} B(\vec{\theta}, \vec{k}, \vec{N})(i, \vec{u}) / \operatorname{det} B(\vec{\theta}, \vec{k}, \vec{N}) \rightarrow 0$ as $\theta_{1} \rightarrow \phi$. Hence, for $\theta_{1}$ sufficiently close to $\phi$, the power series $\tilde{f}(x)=f(x)-g(x)\left(\right.$ or $(f(x)-g(x)) /\left(1-\gamma_{0}\right)$ if $\left.n_{1}=0\right)$ possesses roots of order $k_{j}$ at the $\alpha_{j}$, has lead coefficient one, and has remaining coefficients in $(1+\lambda) I$ for some suitably defined $\lambda>0$, with $\lambda \rightarrow 0$ as $\theta_{1} \rightarrow \phi$. The bound $r(\vec{\theta}, \vec{k} ; I) \leq(1+\lambda) r(\vec{\phi}, \vec{k} ; I)$ then follows as before.

Proof of Lemma 3. Observe that for $m>n>l$ and $\alpha=r e^{2 \pi i \theta}$ the real polynomial

$$
g_{s}(x ; l, m, n, \alpha):=x^{s}\left(x^{l q}-\frac{\epsilon^{n}}{r^{q(n-l)}}\left(\frac{m-l}{m-n}\right) x^{n q}+\frac{\epsilon^{m}}{r^{q(m-l)}}\left(\frac{n-l}{m-n}\right) x^{m q}\right)
$$

satisfies $g(\alpha)=g^{\prime}(\alpha)=0$.

Suppose that $f(x)=1+\sum_{i=1}^{\infty} a_{i} x^{i}$ is a power series in $\mathcal{F}_{I}$ with a double root at $\alpha=r(\theta, 2 ; I) e^{2 \pi i \theta}$. Now if there existed two coefficients with

$$
\left(a_{n q}-B\right) \neq-\epsilon^{n} A, \quad\left(a_{m q}-B\right) \neq \epsilon^{m} A, \quad m>n,
$$

then for a suitably small $\delta>0$ the power series

$$
\tilde{f}(x)=(1-\delta) f(x)+\delta g_{0}\left(x ; 0, m, n, r(\theta, 2 ; I) e^{2 \pi i \theta}\right)
$$

would be an extremal polynomial with all its non-zero coefficients $\tilde{a}_{i}$ strictly inside $I$; which by Lemma 4 cannot occur. Hence the stated form of the coefficients $a_{l q}$.

Now consider an $s \not \equiv 0 \bmod q$. Amongst the extremal power series with a fixed set of coefficients $a_{m}$ for $m \not \equiv s \bmod q$, either there is an extremal series with 
$\left(a_{l q+s}-B\right)=C_{s} \epsilon^{l}$ for all $l$ for some $C_{s}$, or there is some maximal $N$ such that there is a series with

$$
\left(a_{l q+s}-B\right)= \begin{cases}\epsilon^{l} C_{s} & \text { for } 0 \leq l<N, \\ \epsilon^{l} C_{s}^{\prime} & \text { for } l=N\end{cases}
$$

for some $C_{s}^{\prime} \neq C_{s}$. Amongst these series pick the one with $\left|C_{s}^{\prime}-C_{s}\right|$ minimal. We claim that for such a series the remaining coefficients must satisfy $\left(a_{l q+s}-B\right)=$ $\epsilon^{l} \operatorname{sign}\left(C_{s}^{\prime}-C_{s}\right) A$. To see this, observe that if $\left(a_{m q+s}-B\right) \neq \epsilon^{l} \operatorname{sign}\left(C_{s}^{\prime}-C_{s}\right) A$ for some $m>N$, then

$$
\tilde{f}(x)=f(x)+\delta \operatorname{sign}\left(C_{s}^{\prime}-C_{s}\right) \sum_{l=0}^{N-1} g_{s}(x ; l, N, m, \alpha)=1+\sum_{i=0}^{\infty} \tilde{a}_{i} x^{i}
$$

has $\tilde{f}(\alpha)=\tilde{f}^{\prime}(\alpha)=0$ and for sufficiently small $\delta$ it can still have $\tilde{a}_{m q+s}$ inside $I$ and be of the above form with

$$
\begin{gathered}
\tilde{C}_{s}=C_{s}+\delta \operatorname{sign}\left(C_{s}^{\prime}-C_{s}\right), \\
\tilde{C}_{s}^{\prime}=C_{s}^{\prime}-\delta \operatorname{sign}\left(C_{s}^{\prime}-C_{s}\right)\left(\sum_{l=0}^{N-1} \frac{(m-l)}{(m-n) r^{(n-l) q}}\right)
\end{gathered}
$$

satisfying $\left|\tilde{C}_{s}\right|,\left|\tilde{C}_{s}^{\prime}\right| \leq A$ and $\left|\tilde{C}_{s}^{\prime}-\tilde{C}_{s}\right|<\left|C_{s}^{\prime}-C_{s}\right|$, contradicting the assumed minimality.

Proof of Proposition 1. The proof closely resembles the proof of [1, Theorem 1]. First observe [1, Lemma 1] that

$$
F(x):=1+\sum_{i=1}^{k}(-1)^{i} c_{i} x^{m_{i}}, \quad c_{i}:=\prod_{\substack{j=1 \\ j \neq i}}^{k} \frac{m_{j}}{\left|m_{i}-m_{j}\right|},
$$

is a polynomial with a $k$ th order root at 1 . Taking $m_{i}:=i^{2}$, we have

Hence

$$
c_{i}=\frac{2(k !)^{2}}{(k+i) !(k-i) !} \leq 2\left(\frac{k}{k+1}\right)^{i^{2}} \text {. }
$$

$$
g(x):=\frac{1}{s} \sum_{i=1}^{s} F\left(-\left(\frac{k+1}{k}\right)^{b_{i} / b_{s}} x^{b_{i}}\right)
$$

is a polynomial in $\mathcal{F}_{[0, V]}$ with a root of order $k$ at $-(k / k+1)^{1 / b_{s}}$. Similarly, taking $m_{1}:=2, m_{i}:=7(i-1)^{2}, i=2, \ldots, k+1, k \geq 1$, we have

$$
c_{1}=\prod_{j=1}^{k}\left(1-\frac{2}{7 j^{2}}\right)^{-1}<2\left(\frac{k}{k+1}\right)^{2 / 7}
$$

(by induction on $k$ ) and

$$
c_{i+1}=\frac{4}{7 i^{2}-2} \frac{(k !)^{2}}{(k+i) !(k-i) !} \leq \frac{4}{5}\left(\frac{k}{k+1}\right)^{i^{2}}, \quad i \geq 1 .
$$

Hence

$$
g(x):=\frac{1}{s^{\prime}} \sum_{i=1}^{s^{\prime}} F\left(-\left(\frac{k+1}{k}\right)^{b_{i} / 7 b_{s^{\prime}}} x^{b_{i}}\right)
$$

is a polynomial in $\mathcal{F}_{[U, 0]}$ with a root of order $(k+1)$ at $-(k /(k+1))^{1 / 7 b_{s^{\prime}}}$. 
Proof of Proposition 2. Observe that for $A \geq\left(2^{\frac{1}{k}}-1\right)^{-k}$ the power series

$$
\frac{\left(1-\left(A^{\frac{1}{k}}+1\right) x\right)^{k}}{(1-x)}=1-b_{1} x+b_{2} x^{2}-\cdots-(-1)^{k} b_{k-1} x^{k-1}+(-1)^{k} A \frac{x^{k}}{(1-x)}
$$

has a $k$ th order root at $\left(A^{\frac{1}{k}}+1\right)^{-1}$ and, since $\left(A^{\frac{1}{k}}+1\right) \geq k$, coefficients

$$
b_{j}=\sum_{i=0}^{j}(-1)^{j-i}\left(\begin{array}{c}
k \\
i
\end{array}\right)\left(A^{\frac{1}{k}}+1\right)^{i}
$$

satisfying $1<b_{2}<b_{4}<\cdots$ and $0<b_{1}<b_{3}<\cdots$, where $b_{k}=A$ and $b_{k-1}=$ $\left(A^{\frac{1}{k}}+1\right)^{k}-A \leq A$. By Theorem 1 (or, more directly, [1, Theorem 4]) the form of the power series ensures that this gives the smallest root. 


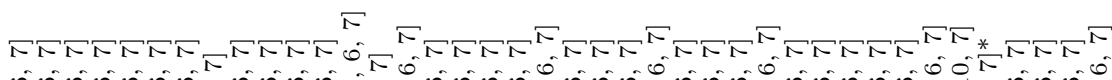

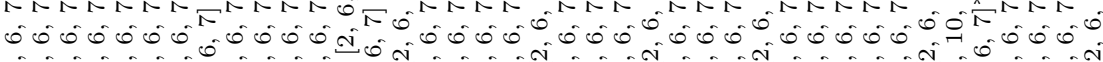

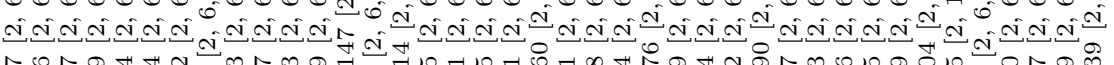

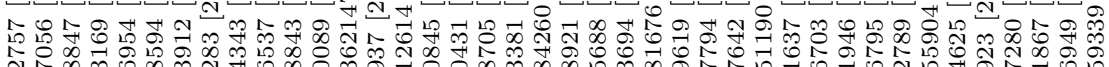

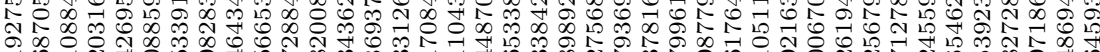

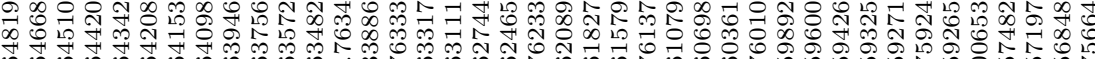

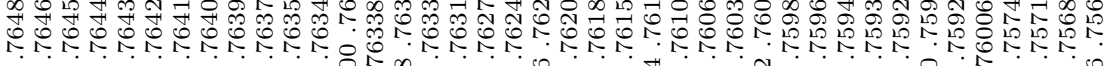

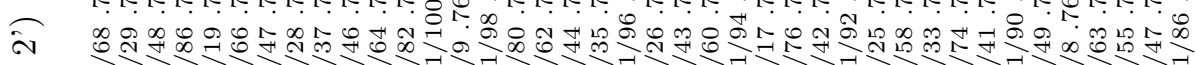

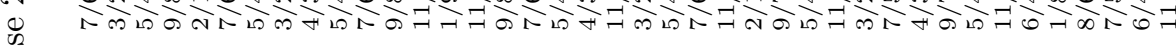

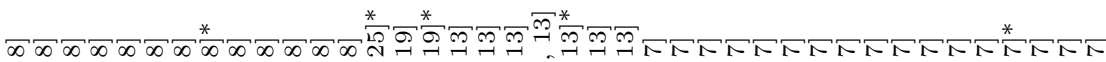


งง

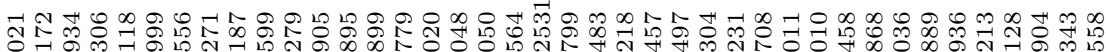

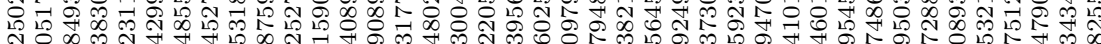

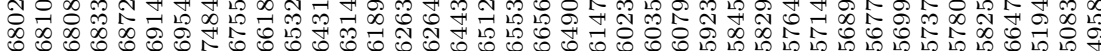

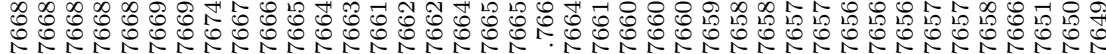
mon लः ता

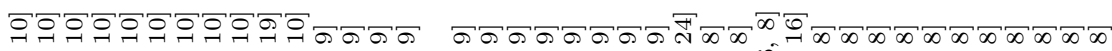

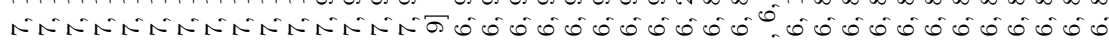

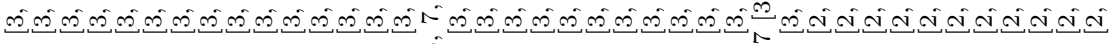

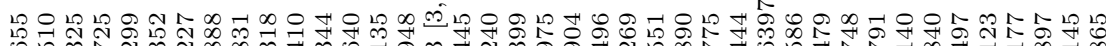

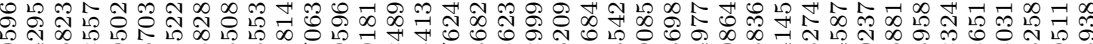

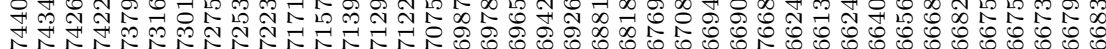

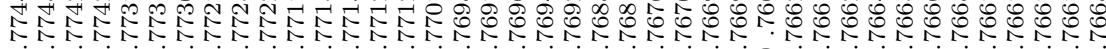

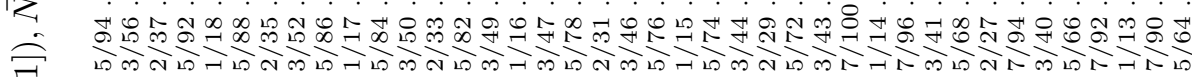

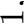

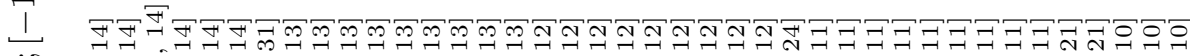

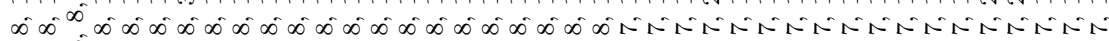

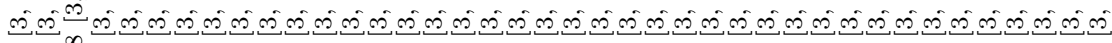



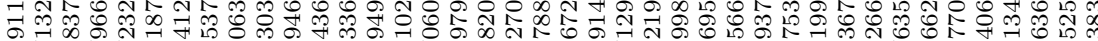

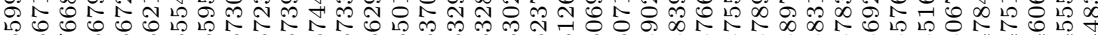

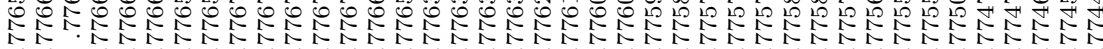

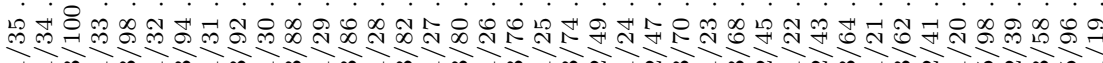

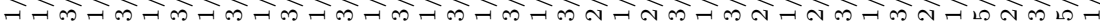

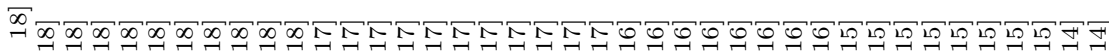

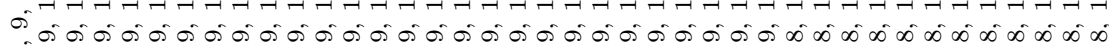

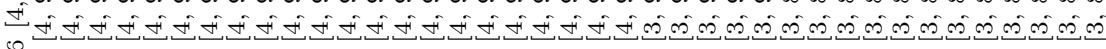

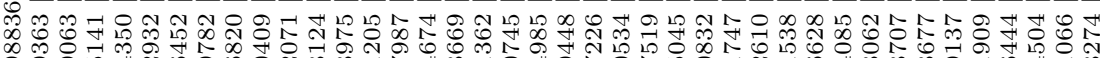

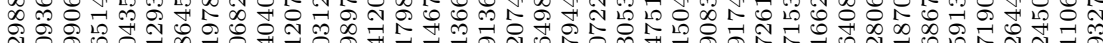



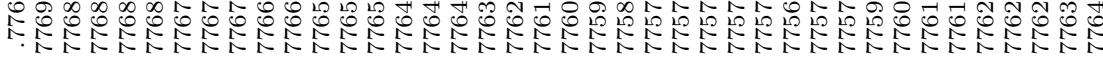
\&o

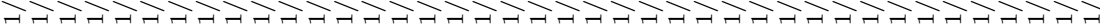




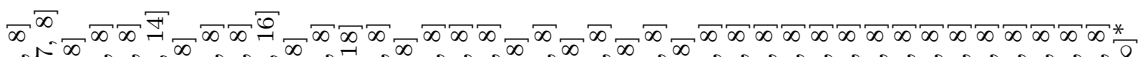

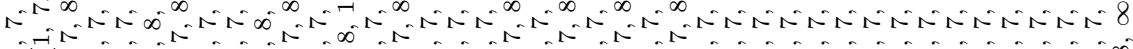

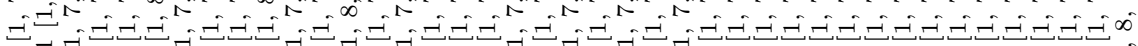

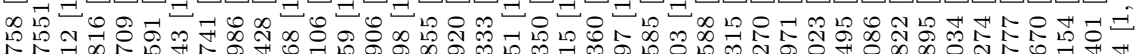

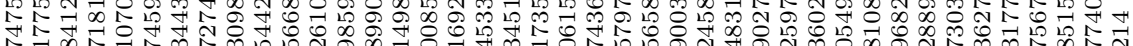

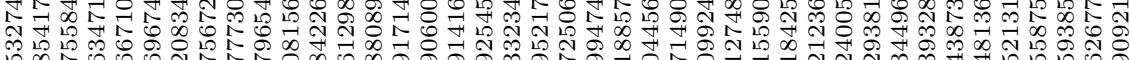

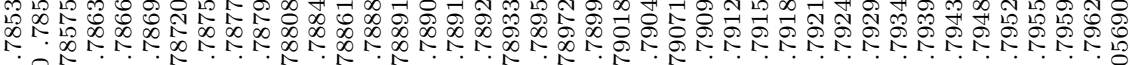

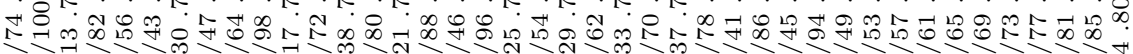
슥

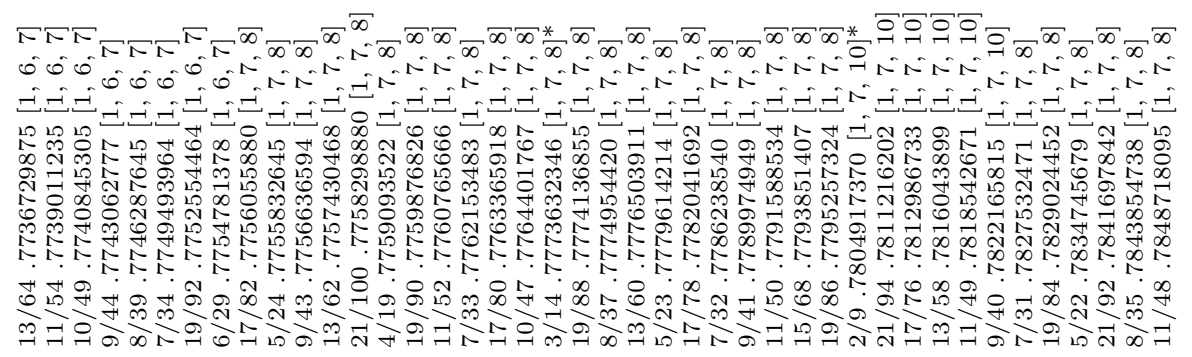

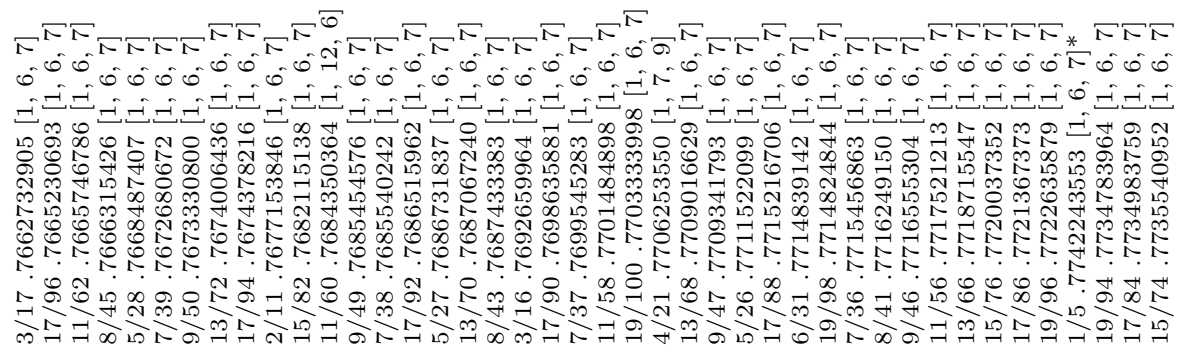



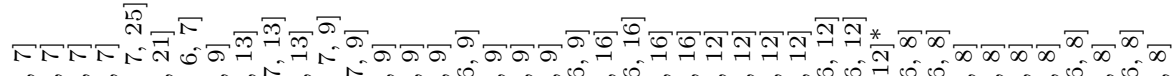
000 O ONANAN 소수



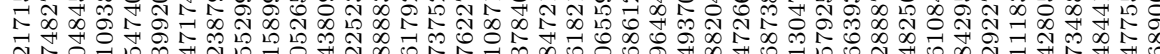

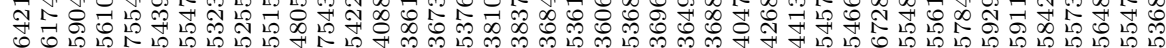

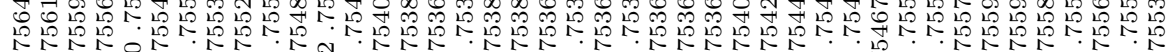
món

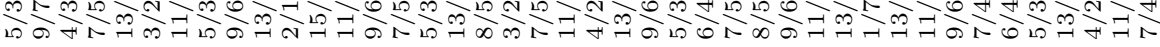




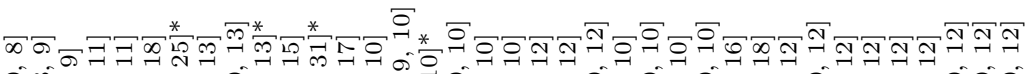

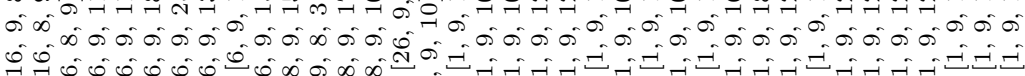

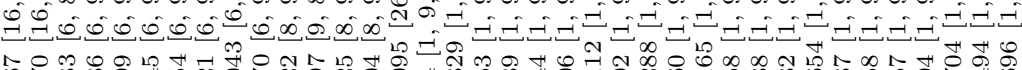
수유.

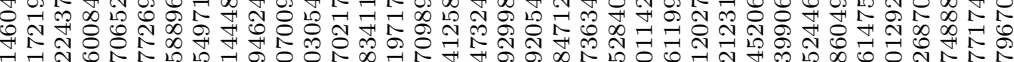

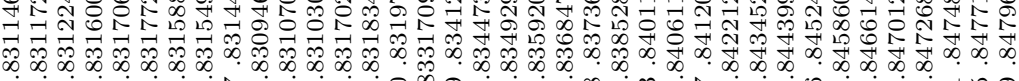



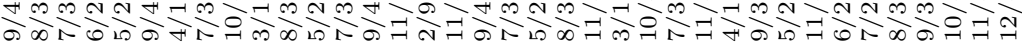



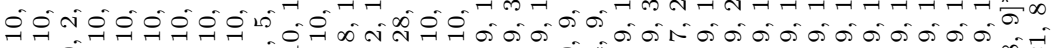

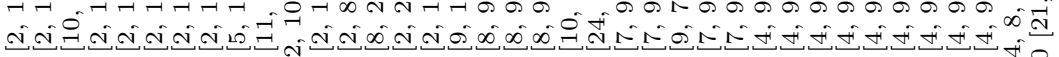
m 至

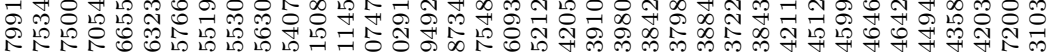

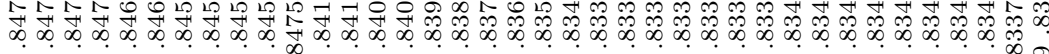

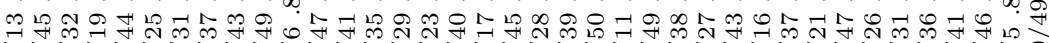
तार

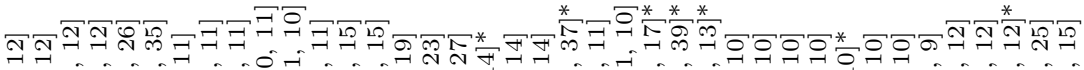



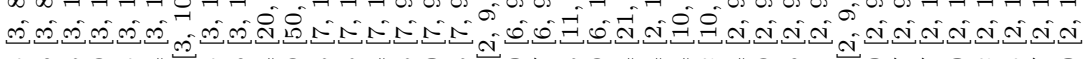

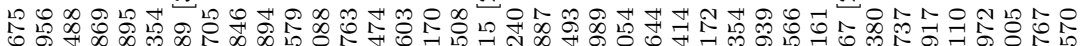

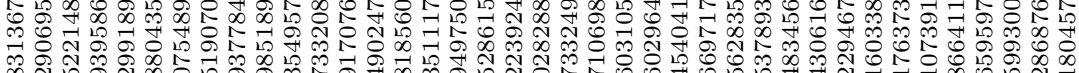
等 急 舟 9 국

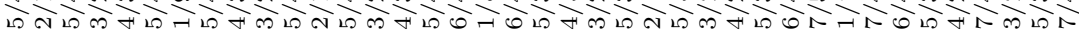

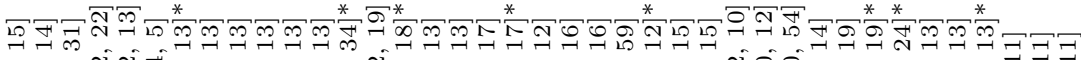

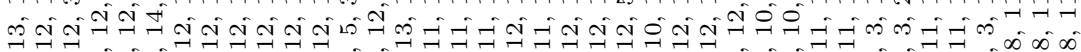

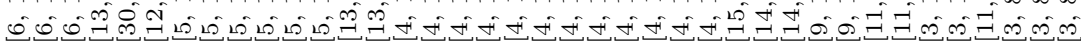

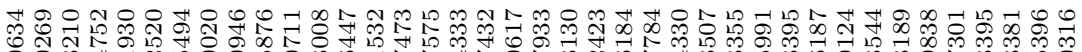

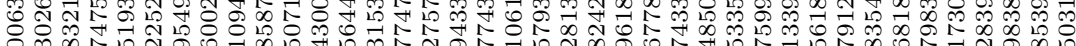

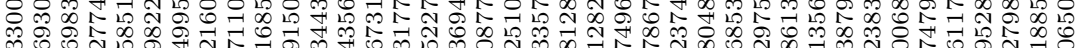

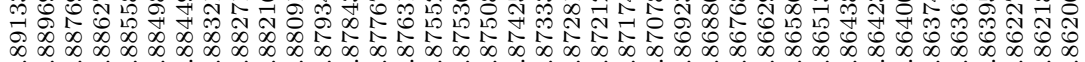

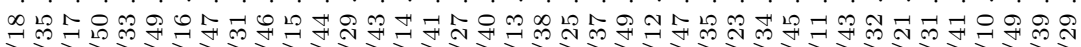

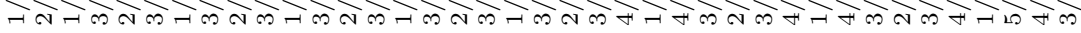

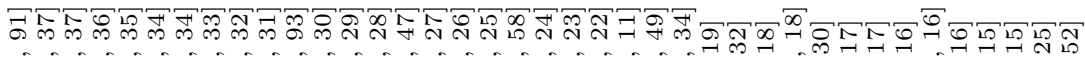

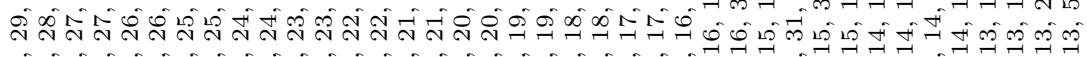

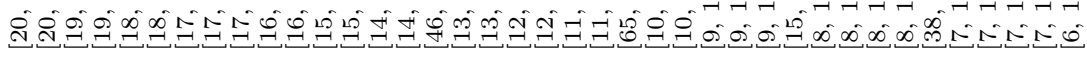

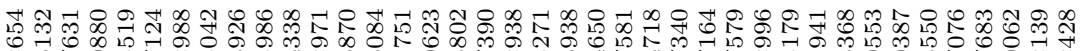

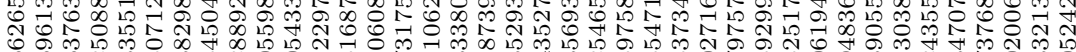

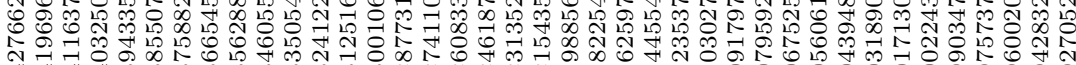

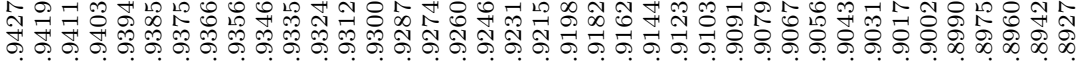

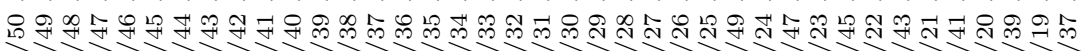

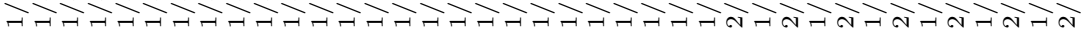




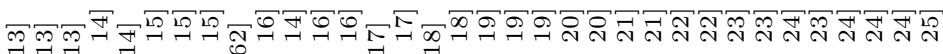
бө。

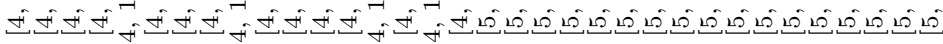

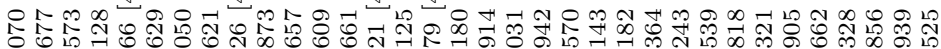

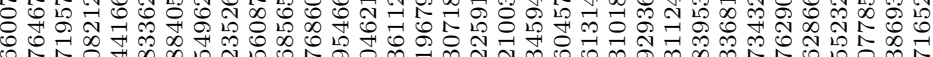
$\begin{array}{lll} & \\ 0 & \end{array}$

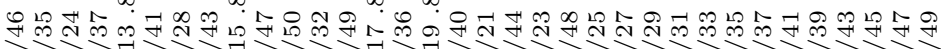

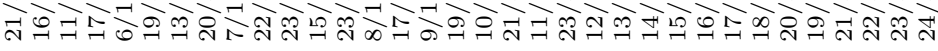

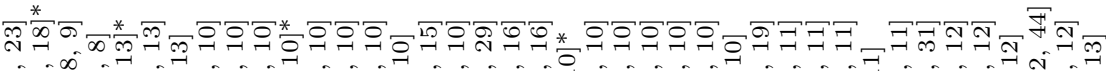

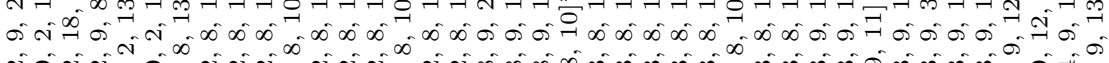

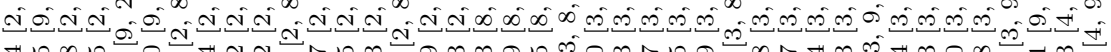

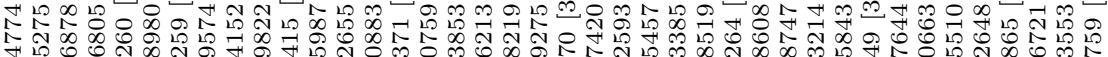

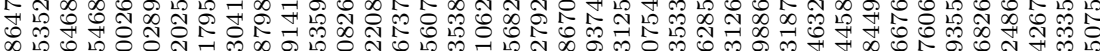

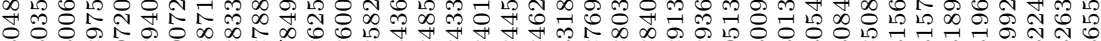

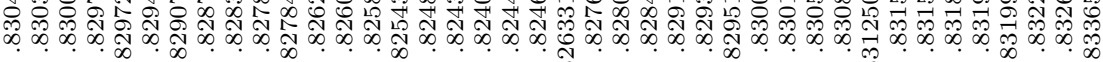

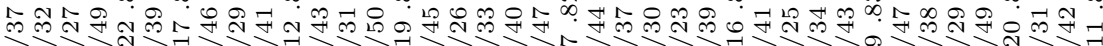

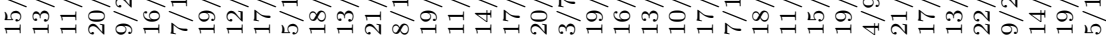

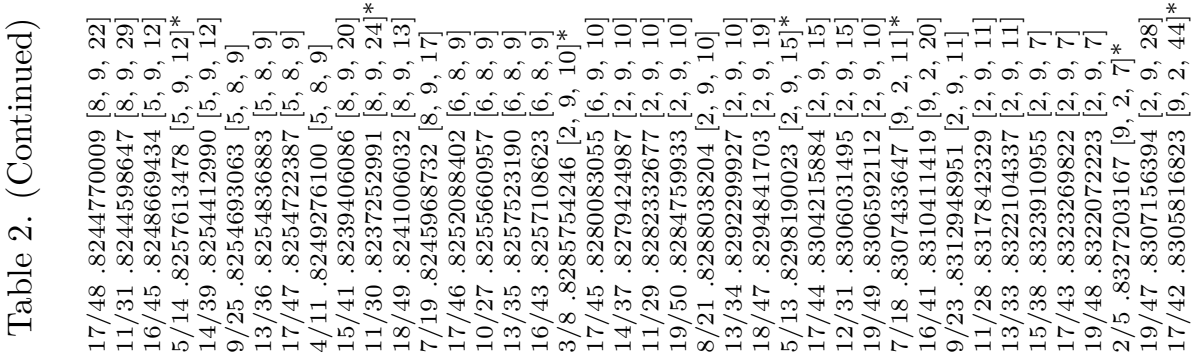

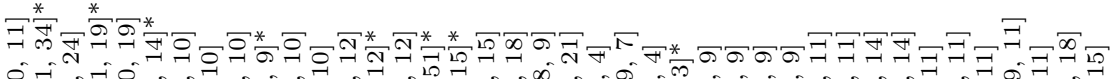

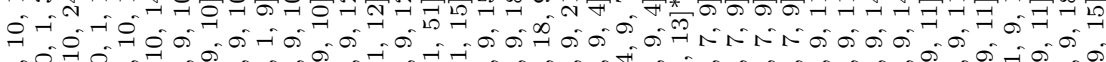

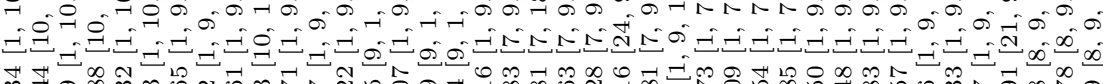
荡。

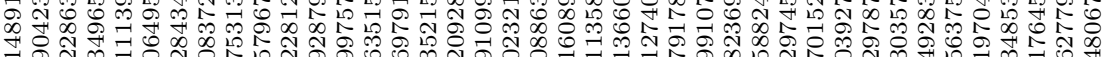

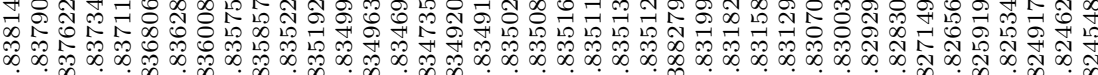

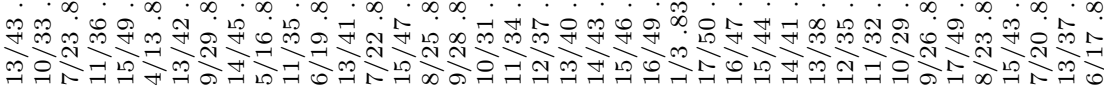

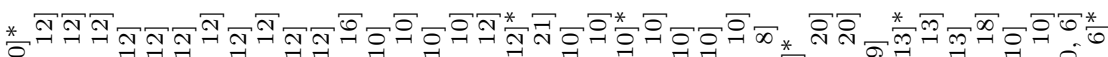

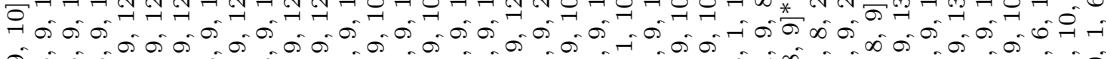

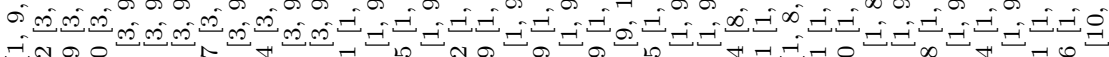

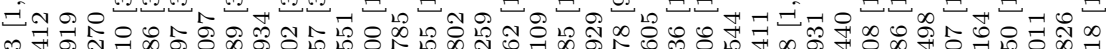

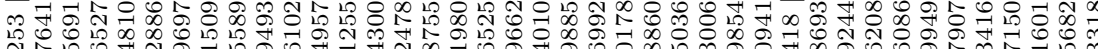

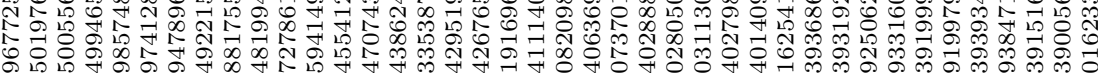

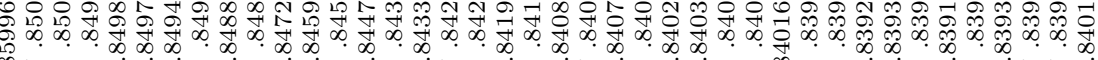

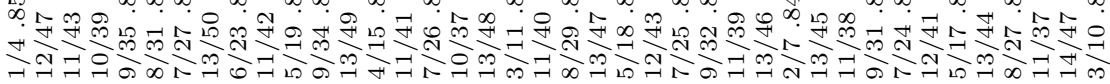




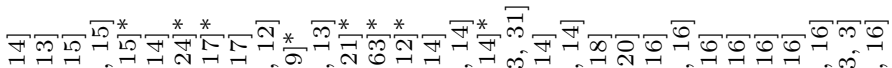

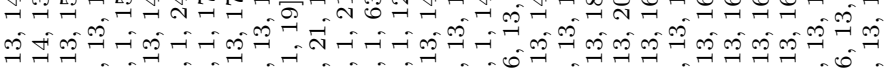

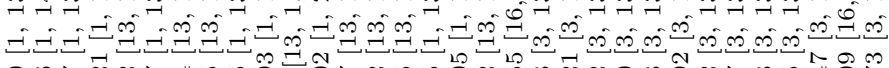
8

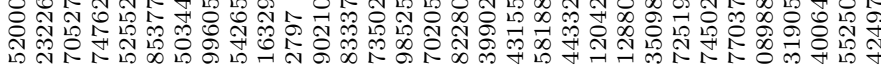

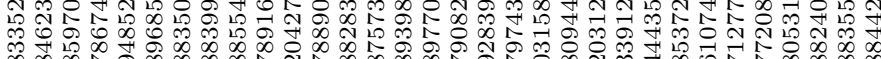

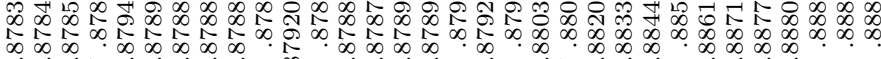

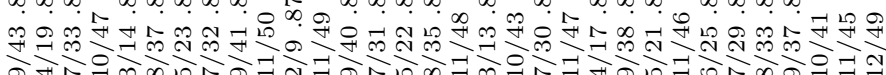



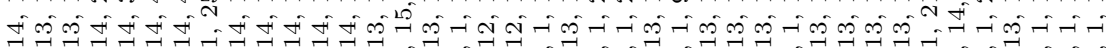

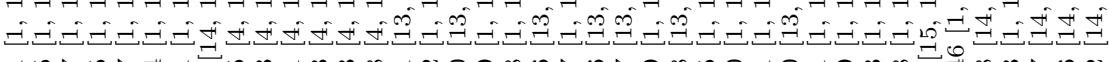

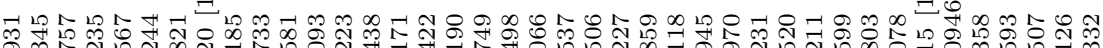

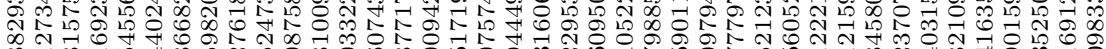

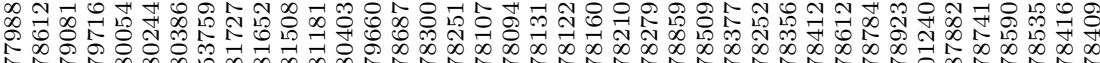

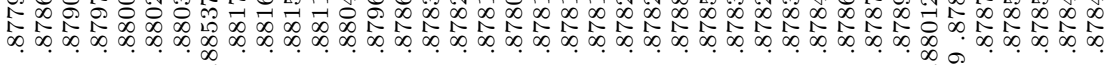

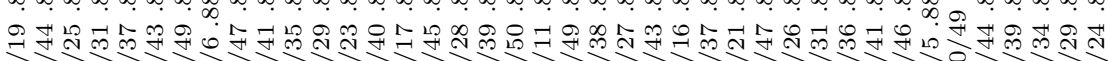
का मूनी

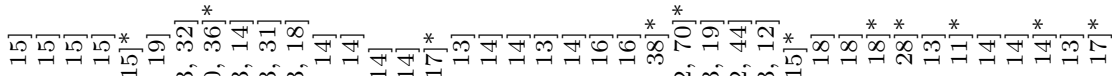
จำง

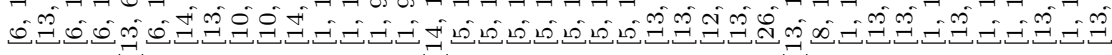
구의

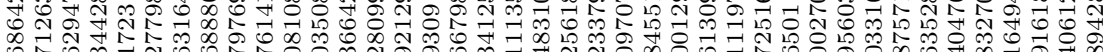

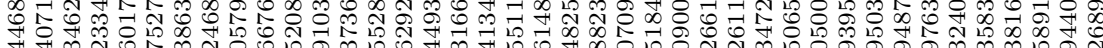

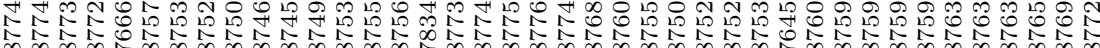

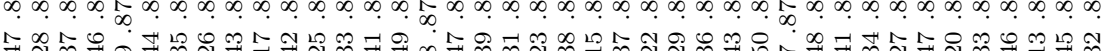

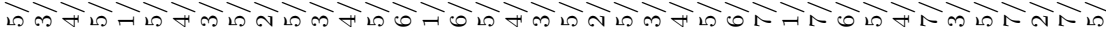

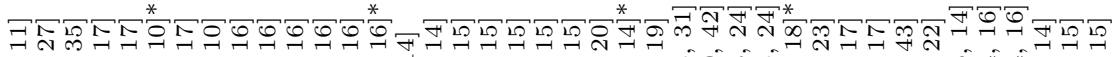

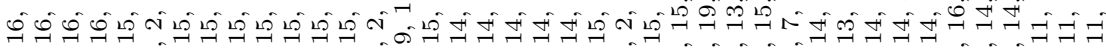

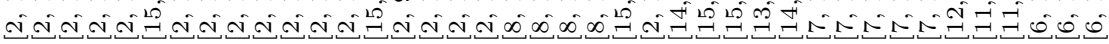



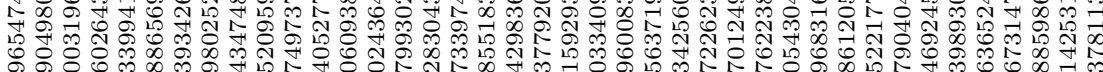



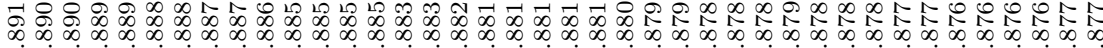

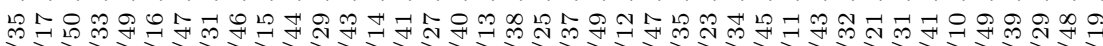



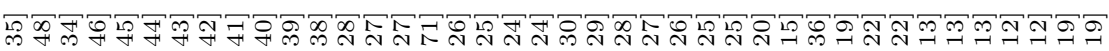

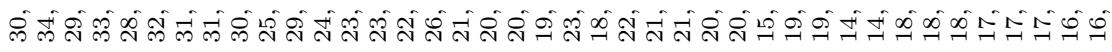

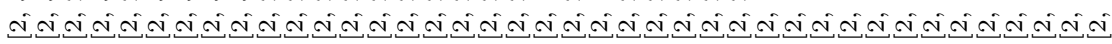

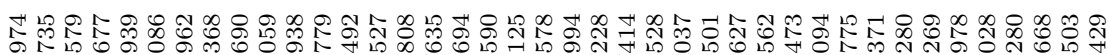

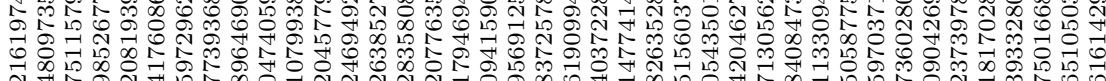

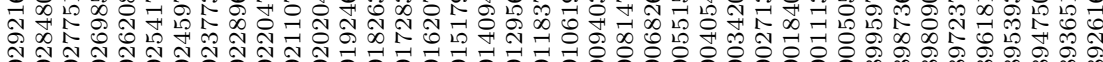



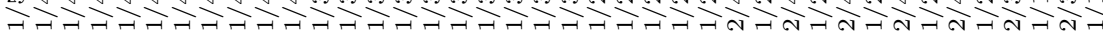




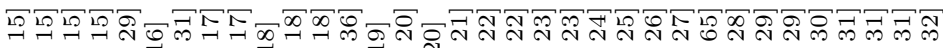

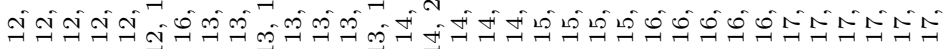
İ

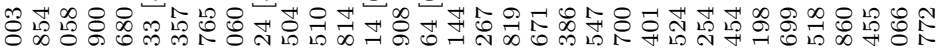

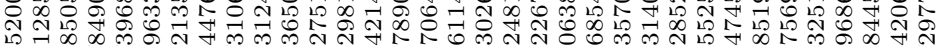

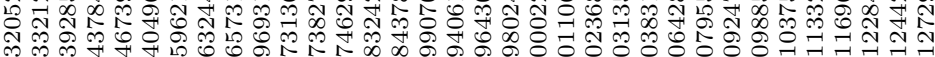

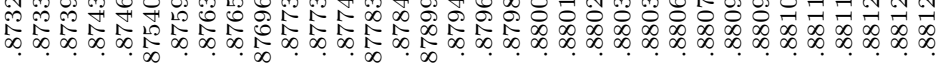

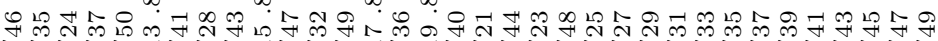

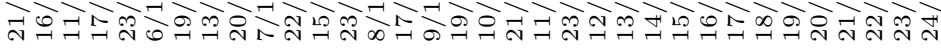

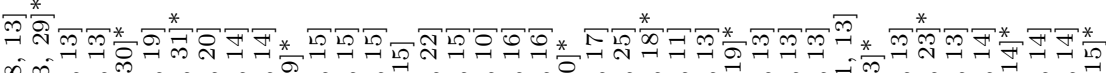

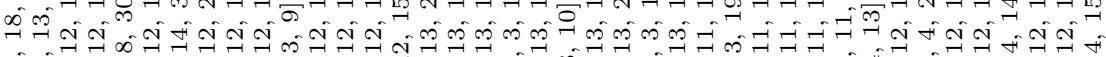

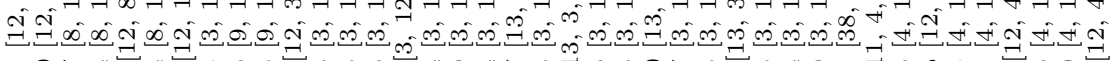

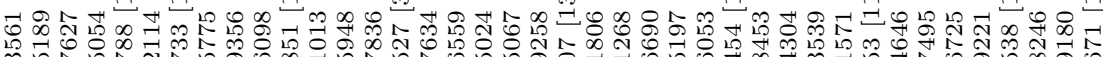

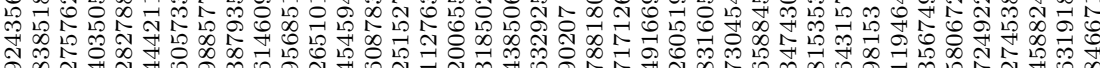
Non



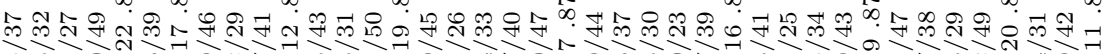

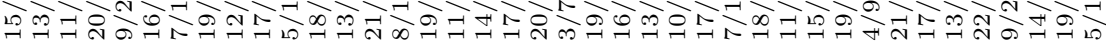

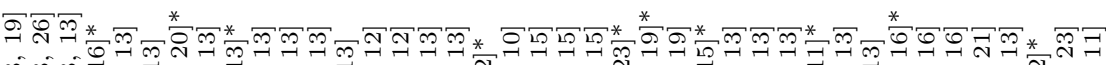
ช.

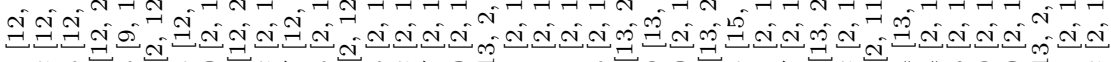

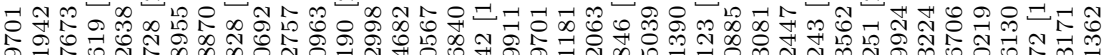

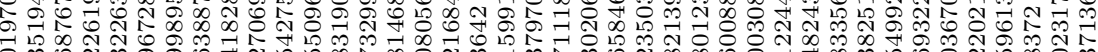

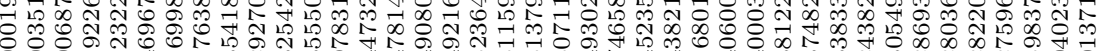

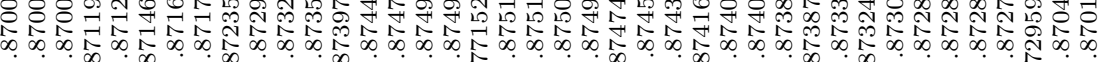

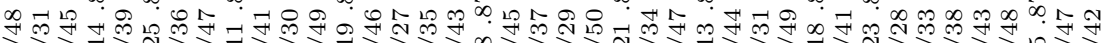

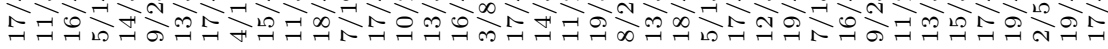

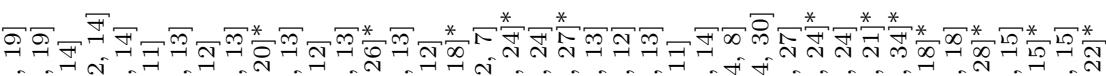
걱

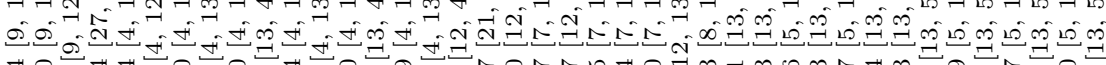

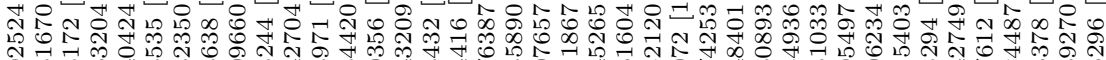

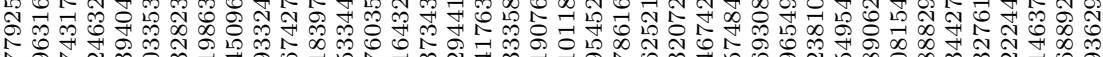

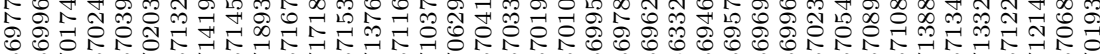

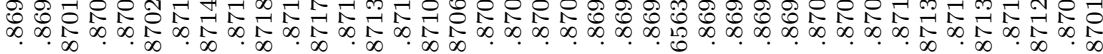

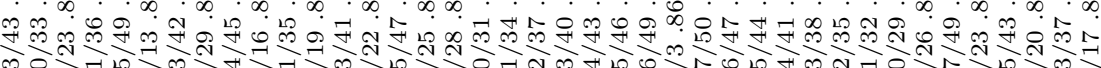

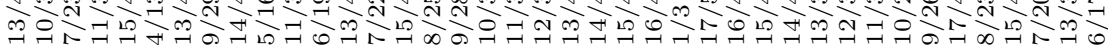

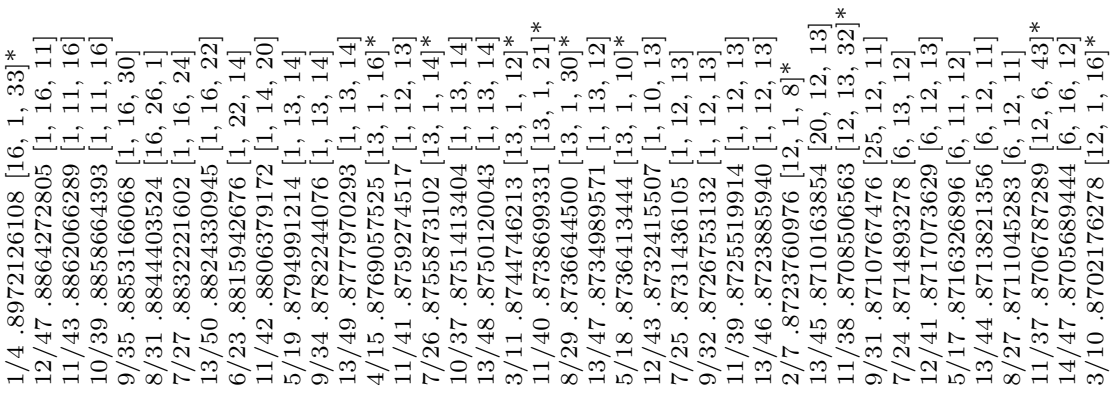




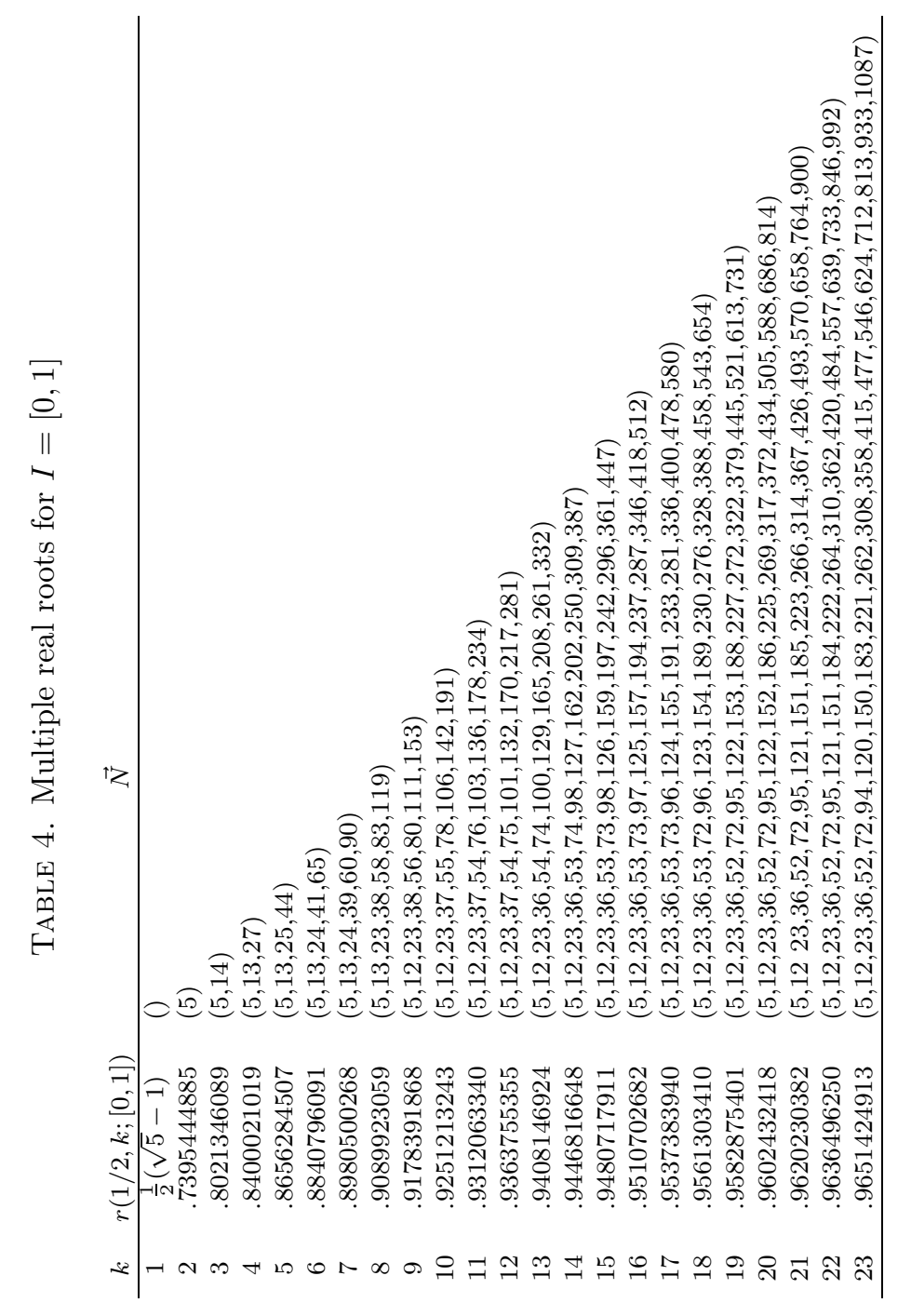




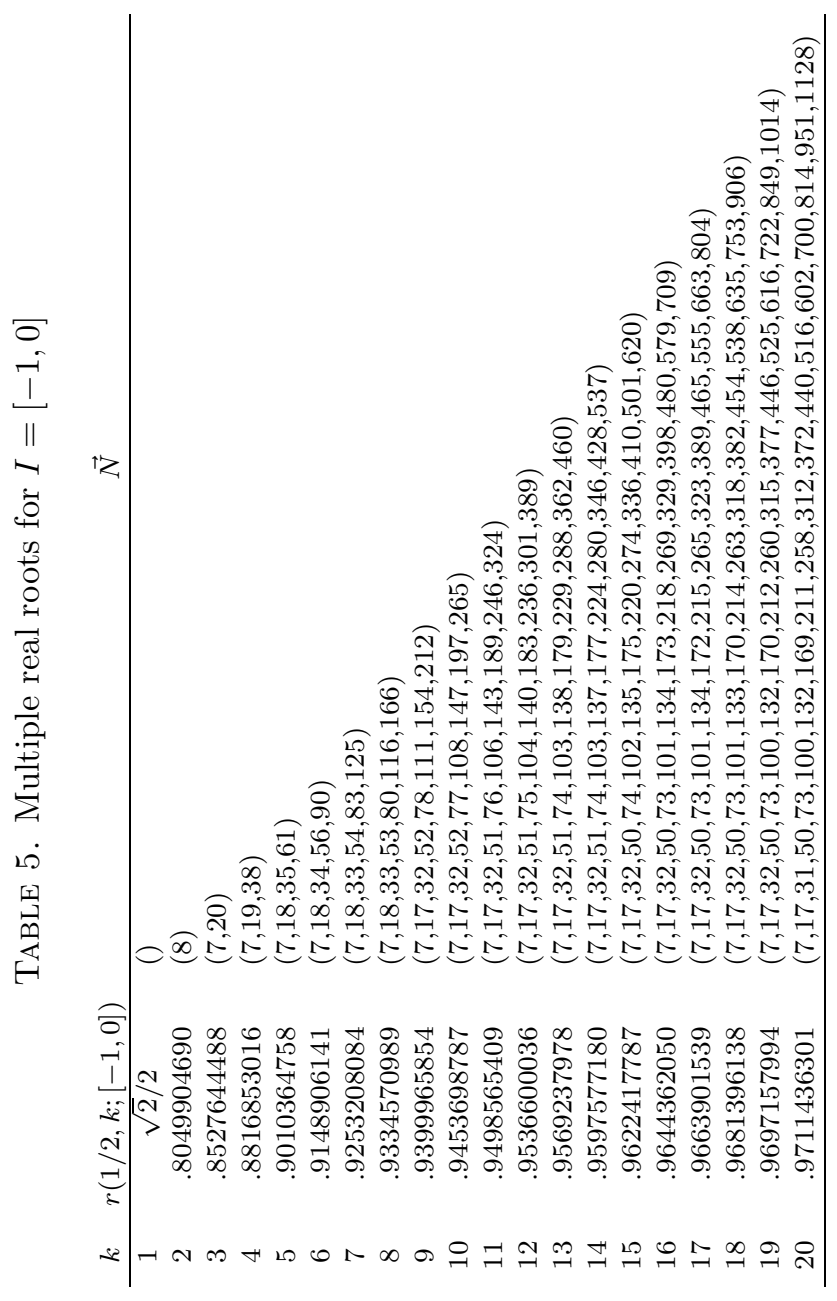




\section{ACKNOWLEDGMENTS}

The author thanks Boris Solomyak for motivating this investigation, and David Boyd and Peter Borwein for their encouragement.

\section{REFERENCES}

[1] F. Beaucoup, P. Borwein, D. W. Boyd \& C. Pinner, Multiple roots of $[-1,1]$ power series, J. London Math. Soc. (2) 57 (1998), 135-147.

[2] F. Beaucoup, P. Borwein, D. W. Boyd \& C. Pinner, Power series with restricted coefficients and a root on a given ray, Math. Comp. 67 (1998), 715-736. CMP 98:07

[3] P. Borwein, T Erdélyi \& G. Kós, Littlewood-type problems on [0,1], Bull. London Math. Soc. to appear.

[4] A. Odlyzko \& B. Poonen, Zeros of polynomials with 0,1 coefficients, L'Enseignement Math. 39 (1993), 317-348. MR 95b:10026

[5] B. Solomyak, Conjugates of beta-numbers and the zero-free domain for a class of analytic functions, Proc. London Math. Soc. (3) 68 (1994), 477-498. MR 95c:30010

[6] B. Solomyak. On the random series $\sum \pm \lambda^{n}$ (an Erdös problem), Ann. Math. 142 (1995), 611-625. MR 97d:11125

Centre for Experimental and Constructive Mathematics, Simon Fraser University, Burnaby, BC V5A 1S6, Canada \& Department of Mathematics, University of British Columbia, Vancouver, BC V6T 1Z2, Canada

Current address: Mathematics and Computer Science, University of Northern British Columbia, 3333 University Way, Prince George, BC V2N 4Z9, Canada

E-mail address: pinnerc@unbc.ca 